\title{
Bose-Einstein-condensation dynamics with a quantum-kinetic approach
}

\author{
O. Morandi, P.-A. Hervieux, and G. Manfredi \\ Institut de Physique et Chimie des Matériaux de Strasbourg, 23 rue du Loess, F-67034 Strasbourg, France
}

(Received 29 April 2013; published 27 August 2013)

\begin{abstract}
The evolution equation of a weakly interacting boson system is derived. The model includes the interaction between the atoms in the condensate and the surrounding gas of noncondensed particles. The Bogoliubov transformation is introduced in a full quantum context and the scattering kernel between dressed particles and the condensate phase is obtained. The final system is expressed by the Boltzmann evolution equation for noncondensed particles coupled to the Gross-Pitaevskii equation for the condensate. We consider an out-ofequilibrium situation that induces a fast production of condensed particles. We apply our model to study the condensation dynamics of positronium atoms by evaporation.
\end{abstract}

DOI: 10.1103/PhysRevA.88.023618

PACS number(s): 67.85.-d, 03.75.Hh, 36.10.Dr, 51.10.+y

\section{INTRODUCTION}

The study of Bose-Einstein condensation has emerged as a multidisciplinary discipline of theoretical and experimental physics. The main concept at the basis of the Bose-Einsteincondensation process is that below a critical temperature, a macroscopic fraction of bosons collapses into the lowestenergy state: the zero-momentum state in free space or the ground state for a system in a confining potential [1]. In the ideal case of a noninteracting boson gas, the critical temperature $T_{c}$ is given by

$$
T_{c}=\frac{2 \pi \hbar^{2}}{m k_{B}}\left(\frac{n}{2.612}\right)^{2 / 3},
$$

where $n$ is the density of the gas, $m$ is the mass of the particles, and $k_{B}$ is the Boltzmann constant. In contrast, no simple and general formulas can be derived for the time necessary to reach the thermodynamical equilibrium and form the condensate. The condensation time can be estimated only by analyzing the details of the cooling process of the gas. The Bose-Einstein-condensation process is usually studied for stable atoms. Consequently, the value of the time necessary to reach the condensed state does not have a strong influence when the condensation experiments are designed.

Since the possibility to create a Bose-Einstein condensate made of positronium has been addressed [2], an increasing effort has been devoted to the study of this metastable atom. A positronium atom consists of an electron and its antiparticle, the positron, bound together. Positronium possesses a mass that is considerably smaller than the typical mass of the atoms usually employed in the condensation experiments. As a consequence, a positronium gas should condensate at a temperature several orders of magnitude greater than the critical temperature of, for example, rubidium. The ground state of positronium consists of triplet states with total spin one and a singlet state with total spin zero. The total angular momentum of the positronium atoms fixes their lifetime and decay channels. The triplet state, orthopositronium, annihilates into three photons and has a mean lifetime of 142 ns. The singlet state, parapositronium, disappears at around $125 \mathrm{ps}$ by emitting two $\gamma$ photons. This short lifetime is a strong limit for the production of a condensate made of positronium. In this contribution, we explore the possibility to accelerate the production of a condensate by considering a strongly out-of-equilibrium situation. We derive the evolution equation of a weakly interacting boson system. The exact low-energy spectra is included in the model. The Bogoliubov transformation is derived in a fully quantum context and the scattering kernel is derived. We model the evaporative cooling process by inserting a cutoff in the energy distribution of a uniform particle gas. The strong nonlinear interaction between condensed and noncondensed particles leads to an efficient and fast collapse of the particles in the ground state.

\section{MODEL}

Here, we derive the evolution equations for the condensed phase and the noncondensed particles of a weakly interacting Bose gas in the quantum-kinetic formalism. The quantumkinetic formalism constitutes a fully quantum framework where the semiclassical description of the particle motion is easily recovered in the limit $\hbar \rightarrow 0$. It displays strong analogies with the classical phase-space description of a statistical system. In this context, inhomogeneity and scattering mechanisms are easily included and quasiequilibrium regimes, such as the hydrodynamic approximation of the diffusive transport, can be naturally obtained. Technically, in our model, the dynamics of weakly interacting bosons is described by combining the quantum Wigner formalism with some general approximations such as the Markovian assumption and the Bogoliubov-Popov description of the pseudoparticles. The collision processes are reproduced by applying the Baliev many-particle theory [3]. In order to develop a consistent formalism, we treat separately the particles in the ground state (forming the condensate) and the quasifree gas of atoms (which constitute a reservoir of noncondensed particles) [4,5]. The evolution equations are obtained by the application of the Bogoliubov transformation and the definition of a suitable set of creation-annihilation operators for quasiparticles. The new particle fields differ from the original ones by the value ( $c$ number) of the wave function of the many-body condensate, usually called the "order parameter." Since the seminal contribution of Bogoliubov, an in-depth investigation of the properties of bosons dressed by the condensate interaction has been performed by various authors. The general properties of the many-body excitation and renormalization procedures are known. In Ref. [6], a clear review of this research field is presented. 
The Boltzmann dynamics in the classical or semiclassical approximation is a well-established framework for the simulation of nonequilibrium phenomena in diluted gas. Dynamical models based on the Boltzmann formalism have been applied to the study of the evolution and thermalization processes of a gas in the supercritical regime. In the field of polariton condensation, the Boltzmann phase-space approach was used to predict many details of the delicate condensation mechanism of this element [7]. Numerical simulations based on the Boltzmann dynamics are also used for the study of the excitation spectra of the condensate $[8,9]$.

The many-body Hamiltonian describing interacting bosons in the presence of an external potential $U$ is given by (see, for example, $[1,10])$

$$
\begin{aligned}
K= & \int \psi^{\dagger}(\mathbf{r})\left[-\frac{\hbar^{2} \Delta}{2 m}+U-\mu\right] \psi(\mathbf{r}) d \mathbf{r} \\
& +\frac{g}{2} \int \psi^{\dagger}(\mathbf{r}) \psi^{\dagger}(\mathbf{r}) \psi(\mathbf{r}) \psi(\mathbf{r}) d \mathbf{r}
\end{aligned}
$$

where $\psi\left(\psi^{\dagger}\right)$ denotes the annihilation (creation) operator of the bosons and $\mu$ is the chemical potential. The elementary particle-particle scattering event is modeled by the hard-sphere interaction with collision strength $g=4 \pi \hbar^{2} a / m$, where $a$ is the scattering length and $m$ is the mass.

In order to develop a convenient mathematical formulation of the problem, it is essential to distinguish between the particles that form the condensate and those belonging to the surrounding gas. The simplest way to proceed is to consider the evolution dynamics of the particles, discarding the quantum-mechanical nature of the condensate. With this simplification, the condensate is the collection of all of the particles with zero momentum. The condensation can be easily described by monitoring the value of the particle distribution function $f(\mathbf{p}, t)$ for $\mathbf{p}=0$ under the macroscopic scaling: $\lim _{N \rightarrow \infty} f(\mathbf{p}=0) / N=n_{0}>0$, where $N$ is the total number of particles of the system. This approach is one of the first methods used to describe the condensation dynamics of polaritons [7,11].

Here, in contrast, we adopt a fully quantum approach. The condensate part of the field operator can be separated from the rest of the particle gas by using the transformation $\psi=\langle\psi\rangle+$ $\widetilde{\psi}$, where the field $\widetilde{\psi}$ satisfies $\langle\widetilde{\psi}\rangle=0$ and $\langle\psi\rangle=\Phi$ is the Bose macroscopic wave function (order parameter) [6]. The bracket $\langle\cdot\rangle$ denotes the expectation value. With this decomposition, the Hamiltonian becomes

$$
\begin{aligned}
K= & \int\left\{\Phi\left(\mathfrak{k}+\frac{g}{2}|\Phi|^{2}\right) \Phi+\left[\left(\mathfrak{k}+g|\Phi|^{2}\right) \bar{\Phi}\right] \widetilde{\psi}+\left[\left(\mathfrak{k}+g|\Phi|^{2}\right) \Phi\right] \widetilde{\psi}^{\dagger}+\widetilde{\psi}^{\dagger}\left(\mathfrak{k}+g|\Phi|^{2}\right) \widetilde{\psi}\right. \\
& \left.+\frac{g}{2}\left(\bar{\Phi}^{2} \widetilde{\psi} \widetilde{\psi}+\Phi^{2} \widetilde{\psi}^{\dagger} \widetilde{\psi}^{\dagger}+2|\Phi|^{2} \widetilde{\psi}^{\dagger} \widetilde{\psi}\right)+g\left(\bar{\Phi} \widetilde{\psi} \tilde{\psi}^{\dagger} \widetilde{\psi} \widetilde{\psi}+\Phi \widetilde{\psi}^{\dagger} \widetilde{\psi}^{\dagger} \widetilde{\psi}+\frac{1}{2} \widetilde{\psi}^{\dagger} \widetilde{\psi}^{\dagger} \widetilde{\psi} \widetilde{\psi}\right)\right\} d \mathbf{r},
\end{aligned}
$$

where an overline denotes conjugation and

$$
\mathfrak{k}=-\frac{\hbar^{2} \Delta}{2 m}+U(\mathbf{r})-\mu .
$$

In this form, the Hamiltonian shows the typical character of a system containing a condensate phase. $K$ contains three-field terms that do not conserve the number of particles and originate from triplet correlation terms of the form $\left\langle\widetilde{\psi}^{\dagger} \widetilde{\psi}^{\dagger} \widetilde{\psi}\right\rangle$. They describe the transfer of an atom into or out of the condensate [12]. Following Ref. [13], we describe the bosonic field in a reference frame that is at rest with respect to the condensate speed $\mathbf{V}_{c}=\frac{\hbar}{m} \nabla_{\mathbf{r}} \varphi_{c}$, where $\varphi_{c}$ is the phase of the order parameter $\Phi=\sqrt{n_{c}} e^{i \varphi_{c}(\mathbf{r}, t)}$. This is obtained by the unitary transformation

$$
\mathcal{U}_{\mathbf{V}_{c}}(t)=e^{-i \int \varphi_{c}(\mathbf{r}, t) \widetilde{\psi}^{\dagger}(\mathbf{r}) \widetilde{\psi}^{\dagger}(\mathbf{r}) d \mathbf{r}} .
$$

The Hamiltonian in the new reference system is

$$
\begin{aligned}
\mathcal{U}_{\mathbf{V}_{c}} K \mathcal{U}_{\mathbf{V}_{c}}^{\dagger}= & \int\left\{\left(\frac{g}{2} n_{c}^{2}+n_{c} m_{c}\right)+\left[m_{c}+g n_{c}^{3 / 2}\right] \widetilde{\psi}^{\dagger}+\left[\overline{m_{c}}+g n_{c}^{3 / 2}\right] \widetilde{\psi}+\widetilde{\psi}^{\dagger}\left[\left(\mathfrak{k}+2 g n_{c}+\frac{m}{2} \mathbf{V}_{c}^{2}\right) \widetilde{\psi}\right]\right. \\
& \left.+\mathbf{V}_{c}\left[\widetilde{\psi}^{\dagger} \overleftrightarrow{j} \widetilde{\psi}\right]+\frac{g}{2} n_{c}\left(\widetilde{\psi} \widetilde{\psi}+\widetilde{\psi}^{\dagger} \widetilde{\psi}^{\dagger}\right)+g \sqrt{n_{c}}\left(\widetilde{\psi}^{\dagger} \widetilde{\psi} \widetilde{\psi}+\widetilde{\psi}^{\dagger} \widetilde{\psi}^{\dagger} \widetilde{\psi}\right)+\frac{g}{2} \widetilde{\psi}^{\dagger} \widetilde{\psi}^{\dagger} \widetilde{\psi} \widetilde{\psi}\right\} d \mathbf{r},
\end{aligned}
$$

where we defined

$$
\begin{aligned}
m_{c} & \equiv-\frac{\hbar^{2}}{2 m}\left[\Delta \sqrt{n_{c}}+i 2 \nabla \varphi_{c} \nabla \sqrt{n_{c}}+i \Delta \varphi_{c} \sqrt{n_{c}}-\sqrt{n_{c}}\left(\nabla \varphi_{c}\right)^{2}\right]+\sqrt{n_{c}}(U-\mu), \\
\widetilde{\psi}^{\dagger}(\mathbf{r}) \overleftrightarrow{j} \widetilde{\psi}(\mathbf{r}) & \equiv \frac{i \hbar}{2}\left\{\left[\nabla \widetilde{\psi}^{\dagger}(\mathbf{r})\right] \widetilde{\psi}(\mathbf{r})-\widetilde{\psi}^{\dagger}(\mathbf{r})[\nabla \widetilde{\psi}(\mathbf{r})]\right\} .
\end{aligned}
$$

Following Ref. [6], we adopt the so-called zero-gap approximation $m_{c} \equiv-g n_{c}^{3 / 2}$. 


\section{NONCONDENSATE GAS: EQUATION OF MOTION}

In this section, we derive the evolution equation for the noncondensed part of the boson gas. The system is described by the following $2 \times 2$ density matrix:

$$
\begin{aligned}
\hat{\rho}\left(\mathbf{r}, \mathbf{r}^{\prime}, t, t^{\prime}\right) & =\Psi(\mathbf{r}, t)\left[\Psi^{\dagger}\left(\mathbf{r}^{\prime}, t^{\prime}\right)\right]^{t} \\
& =\left(\begin{array}{cc}
\widetilde{\psi}(\mathbf{r}, t) \widetilde{\psi}^{\dagger}\left(\mathbf{r}^{\prime}, t^{\prime}\right) & \widetilde{\psi}(\mathbf{r}, t) \widetilde{\psi}\left(\mathbf{r}^{\prime}, t^{\prime}\right) \\
\widetilde{\psi}^{\dagger}(\mathbf{r}, t) \widetilde{\psi}^{\dagger}\left(\mathbf{r}^{\prime}, t^{\prime}\right) & \widetilde{\psi}^{\dagger}(\mathbf{r}, t) \widetilde{\psi}\left(\mathbf{r}^{\prime}, t^{\prime}\right)
\end{array}\right),
\end{aligned}
$$

where we introduce the compact notation $\Psi=\left(\begin{array}{c}\widetilde{\psi} \\ \widetilde{\psi}^{\dagger}\end{array}\right)$. The form of the density matrix given by Eq. (2) is quite unusual for the description of the dynamics of an atomic gas. Since the nondiagonal terms do not conserve the total number of particles, they vanish for every statistical ensemble of eigenstates of the number operator. This is the standard situation for the large majority of systems that can be encountered in solid-state or plasma physics. The presence of a condensed phase induces strong correlations among particles with small momenta. The ground state is a coherent quantum superposition of states with different occupation numbers. This makes the analysis of the particle motion more difficult. The presence of the two-field terms $\widetilde{\psi} \widetilde{\psi}, \widetilde{\psi}^{\dagger} \widetilde{\psi}^{\dagger}$ generates a quite impressive proliferation of Feynman diagrams. They are generally denoted "anomalous" terms. Moreover, the $2 \times 2$ matrix form of the density $\hat{\rho}$ introduces two dynamical variables (the diagonal elements are the same except for a commutation) $\langle\widetilde{\psi} \widetilde{\psi}\rangle,\left\langle\widetilde{\psi}^{\dagger} \widetilde{\psi}^{\dagger}\right\rangle$ whose presence modifies the evolution of the usual probability density $\left\langle\widetilde{\psi}^{\dagger} \widetilde{\psi}\right\rangle$. The exact evolution equation for $\hat{\rho}$ is generally hard to solve. The technical difficulties can be considerably reduced if the density matrix is always maintained approximatively diagonal. This can be done by exploiting the Bogoliubov transformation. We introduce the Green function

$$
\widehat{\mathcal{G}}\left(\mathbf{r}, \mathbf{r}^{\prime} ; t, t^{\prime}\right)=-i \mathcal{T}\left\{\Psi(\mathbf{r}, t)\left[\Psi^{\dagger}\left(\mathbf{r}^{\prime}, t^{\prime}\right)\right]^{t}\right\},
$$

where $\mathcal{T}$ denotes the temporal ordering operator. The evolution equation for $\mathcal{G}$ is

$$
\sigma_{z} \widehat{\mathfrak{H}}_{t}\left\langle\widehat{\mathcal{G}}\left(\mathbf{r}, t ; \mathbf{r}^{\prime}, t^{\prime}\right)\right\rangle=\hbar \delta\left(t-t^{\prime}\right) \delta\left(\mathbf{r}-\mathbf{r}^{\prime}\right)+\langle\mathfrak{S} \widehat{\mathcal{G}}\rangle,
$$

where we defined $\widehat{\mathfrak{H}}_{t}=i \hbar \frac{\partial}{\partial t}-\sigma_{z} \widehat{\mathfrak{H}}(\mathbf{r}, t)$ and

$$
\widehat{\mathfrak{H}}(\mathbf{r}, t)=\left(\begin{array}{cc}
\widehat{\mathfrak{h}} & g n_{c} \\
g n_{c} & \widehat{\mathfrak{h}}
\end{array}\right)
$$

with

$$
\begin{aligned}
\widehat{\mathfrak{h}}= & \frac{1}{2 m}\left[-i \hbar \nabla_{\mathbf{r}}+m \mathbf{V}_{c}(\mathbf{r}, t)\right]^{2}+U(\mathbf{r})-\mu \\
& +2 g n_{c}(\mathbf{r}, t)+2 g n(\mathbf{r}, t) .
\end{aligned}
$$

Here, $\sigma_{z}$ denotes the spin Pauli matrix $\left(\begin{array}{cc}1 & 0 \\ 0 & -1\end{array}\right), \delta$ is the Dirac's delta function, and $\mathfrak{S}$ is the self-energy operator. The last term of Eq. (6) is the Hartree-Fock mean field contribution of the noncondensed particles ( $n$ denotes the density of the gas). The explicit form of $\mathfrak{S}$ is given in Appendix B.

Below the critical temperature, the excitation spectra is more conveniently expressed by the quasiparticle energies rather than the bare-particle energies $[6,14]$. Expressing the equation of motion in terms of the Bogoliubov quasiparticles leads to several technical difficulties. In Refs. [14,15], the spectral density is written in terms of the eigenfunctions of the $2 \times 2$ Hamiltonian given in Eq. (5). The quasiparticle approximation (which states that the Bogoliubov excitations have a infinite lifetime) is adopted. This method is exact only for uniform systems or, equivalently, up to the zeroth order in the Planck constant $\hbar$. Here, we adopt a different strategy. We express the evolution of the system in the quantum phase-space formalism developed by Wigner and Weyl. We perform a unitary rotation of the original representation that can systematically be developed in terms of $\hbar$. This method has already been applied to other systems $[16,17]$. In particular, we recover the Bogoliubov transformation at the leading order of the $\hbar$ expansion. For the development of the theory, it is useful to write the equations of motion in a formalism where the time and space variables are treated symmetrically. We consider the following two-time Wigner-Weyl transformation:

$$
\begin{aligned}
& \mathcal{G}(\mathbf{R}, \mathbf{p} ; T, \omega) \\
& \equiv \mathcal{W}^{-1}\left[\widehat{\mathcal{G}}\left(\mathbf{r}, t ; \mathbf{r}^{\prime}, t^{\prime}\right)\right](\mathbf{R}, \mathbf{p} ; T, \omega) \\
&= \frac{1}{(2 \pi)^{4}} \int \widehat{\mathcal{G}}\left(\mathbf{R}+\frac{\hbar \boldsymbol{\eta}}{2}, T+\frac{\hbar \tau}{2} ; \mathbf{R}-\frac{\hbar \boldsymbol{\eta}}{2}, T-\frac{\hbar \tau}{2}\right) \\
& \times e^{-i(\mathbf{p} \cdot \boldsymbol{\eta}-\tau \omega)} d \boldsymbol{\eta} d \tau .
\end{aligned}
$$

The Wigner-Weyl quantization procedure provides a convenient representation of a generic quantum-mechanical operator by a function defined in the phase space. This is an intermediary situation in between the standard position or momentum representation of the density matrix. One of the main advantages of introducing the Wigner-Weyl map [Eq. (7)] is the availability of the so-called Moyal product $\star$,

$$
\begin{aligned}
\mathcal{W}^{-1}[\widehat{\mathcal{A}} \widehat{\mathcal{B}}](\mathbf{r}, \mathbf{p} ; T, \omega) & \equiv \mathcal{A} \star \mathcal{B} \\
& =\mathcal{A} e^{\frac{i \hbar}{2}\left(\overleftarrow{\nabla_{\mathbf{r}}} \cdot \overrightarrow{\nabla_{\mathbf{p}}}-\overleftarrow{\nabla_{\mathbf{p}}} \cdot \overrightarrow{\nabla_{\mathbf{r}}}\right)-\frac{i \hbar}{2}\left(\overleftarrow{\partial_{T}} \cdot \overrightarrow{\partial_{\omega}}-\overleftarrow{\partial_{\omega}} \overrightarrow{\partial_{T}}\right)} \mathcal{B}
\end{aligned}
$$

Here, $\widehat{\mathcal{A}}, \widehat{\mathcal{B}}$ are quantum-mechanical operators and we used the notation $\mathcal{A}(\mathbf{r}, \mathbf{p}) \equiv \mathcal{W}^{-1}[\widehat{\mathcal{A}}]$. The exponential expressions contained in Eq. (8) have the meaning of $\hbar$ expansion,

$$
\begin{aligned}
& \mathcal{A} e^{\frac{i \hbar}{2}\left(\overleftarrow{\nabla_{\mathbf{r}}} \cdot \overrightarrow{\nabla_{\mathbf{p}}}-\overleftarrow{\nabla_{\mathbf{p}}} \cdot \vec{\nabla}_{\mathbf{r}}\right)} \mathcal{B} \\
& =\sum_{n}\left(\frac{i \hbar}{2}\right)^{n} \frac{1}{n !} \mathcal{A}(\mathbf{r}, \mathbf{p})\left[\overleftarrow{\nabla_{\mathbf{r}}} \cdot \overrightarrow{\nabla_{\mathbf{p}}}-\overleftarrow{\nabla_{\mathbf{p}}} \cdot \overrightarrow{\nabla_{\mathbf{r}}}\right]^{n} \mathcal{B}(\mathbf{r}, \mathbf{p})
\end{aligned}
$$

The arrows indicate the operators on which the derivatives act (a similar expression holds for the time-energy term). The Moyal representation of the Hamiltonian operator is

$$
\begin{aligned}
\mathfrak{H}_{t}= & \mathcal{W}^{-1}\left\{\widehat{\mathfrak{H}}_{t}\right\}=\omega-\mathbf{p} \cdot \mathbf{V}_{c}(\mathbf{r}, t) \\
& -\left(\begin{array}{rr}
\mathfrak{e}(\mathbf{r}, \mathbf{p}, t) & -g n_{c}(\mathbf{r}, t) \\
g n_{c}(\mathbf{r}, t) & -\mathfrak{e}(\mathbf{r}, \mathbf{p}, t)
\end{array}\right),
\end{aligned}
$$

where we defined

$$
\begin{aligned}
\mathfrak{e}(\mathbf{r}, \mathbf{p}, t)= & \frac{\mathbf{p}^{2}}{2 m}+\frac{1}{2} m \mathbf{V}_{c}^{2}(\mathbf{r}, t)+U(\mathbf{r})-\mu \\
& +2 g n_{c}(\mathbf{r}, t)+2 g n(\mathbf{r}, t) .
\end{aligned}
$$

From Eq. (4), it follows that

$$
\left[\widehat{\mathfrak{H}}_{t},\left\langle\widehat{\mathcal{G}} \sigma_{z}\right\rangle\right]=\left\langle\sigma_{z} \mathfrak{S} \widehat{\mathcal{G}} \sigma_{z}\right\rangle-\langle\widehat{\mathcal{G}} \mathfrak{S}\rangle,
$$

where the square brackets denote commutation. We express the previous equation on the Bogoliubov basis by multiplying 
the evolution equation with a suitable unitary operator $\widehat{\Theta}$ (a similar projection procedure is explained in detail in [17]). We multiply Eq. (11) by $\widehat{\Theta}$ (left) and $\widehat{\Theta}^{-1}$ (right). We denote by $\mathcal{K}$ the Hamiltonian written in the Bogoliubov basis,

$$
\mathcal{K}(\mathbf{r}, \mathbf{p}, t)=\mathcal{W}^{-1}\left\{\widehat{\Theta}_{\mathfrak{H}_{t}} \widehat{\Theta}^{-1}\right\}=\Theta \star \mathfrak{H}_{t} \star \Theta^{-1}
$$

The application of the Wigner-Weyl transform to Eq. (11) gives

$$
\begin{aligned}
\mathcal{W}^{-1}\left\{\left[\widehat{\Theta} \widehat{\mathfrak{H}}_{t} \widehat{\Theta}^{-1}, \widehat{G}\right]\right\}= & {[\mathcal{K}, G]_{\star} } \\
= & \mathcal{W}^{-1}\left\{\widehat{\Theta}\left\langle\sigma_{z} \mathfrak{S} \widehat{\Theta}^{-1} \widehat{G}\right\rangle\right. \\
& \left.-\left\langle\widehat{G} \widehat{\Theta} \sigma_{z} \mathfrak{S}\right\rangle \widehat{\Theta}^{-1}\right\}
\end{aligned}
$$

where we defined $G=\Theta \star\langle\mathcal{G}\rangle \star \sigma_{z} \Theta^{-1}$ and the subscript $\star$ indicates that the commutators are written in the algebra of the Moyal product $[\mathcal{A}, \mathcal{B}]_{\star}=\mathcal{A} \star \mathcal{B}-\mathcal{B} \star \mathcal{A}$. The matrix $\Theta$ defines the reference used to describe the system. It is convenient to fix $\Theta$ such that the Hamiltonian symbol becomes diagonal in the classical limit $\hbar \rightarrow 0$. Explicitly, $\Theta$ is written

$$
\Theta=\frac{1}{\sqrt{2 \mathfrak{E}}}\left(\begin{array}{cc}
\sqrt{\mathfrak{E}+\mathfrak{e}} & \sqrt{\mathfrak{E}-\mathfrak{e}} \\
-\sqrt{\mathfrak{E}-\mathfrak{e}} & -\sqrt{\mathfrak{E}+\mathfrak{e}}
\end{array}\right),
$$

with

$$
\mathfrak{E}=\sqrt{\mathfrak{e}^{2}-g^{2} n_{c}^{2}} .
$$

In this way, $\Theta \mathfrak{H}_{t} \Theta^{-1}=\omega-\mathbf{p} \cdot \mathbf{V}_{c}-\sigma_{z} \mathfrak{E}$. Equation (12) provides the evolution of the noncondensed particles at all orders in $\hbar$ (fully quantum). As explained in Appendix A, the $\Theta$ transformation extends the Bogoliubov definition of quasiparticle to higher orders in $\hbar$. The equation of motion is displayed in a compact way in Eq. (12). Explicitly, it can be written in a integro-differential form which is quite hard (but not impossible; see, for example, [18] for a similar system) to solve numerically. Anyway, in many cases, quantization effects are not dominant. In the present work, we will limit ourselves to derive the evolution equation at zeroth order in $\hbar$. We remark that the use of the Wigner-Weyl formalism allows one to maintain a strict control of the approximations: the $\hbar$ expansion is a systematic procedure that we apply to an evolution system that is, in principle, exact (compare with the analogous systems derived in [14,15]).

The unknown of the evolution equation (12) is the Green function, $G(\mathbf{R}, \mathbf{p} ; T, \omega)$. It is a $2 \times 2$ matrix whose variables are the mean position $\mathbf{R}$, the momentum $\mathbf{p}$, the mean time $T$, and the energy variable $\omega$. The latter arises from the Fourier transformation which was performed on the time variables of the field operators [see the definition given in Eq. (7)]. The operators $\psi^{\dagger}(t) \psi\left(t^{\prime}\right)$ describe the temporal correlation between two positions or momenta and the Fourier transform of the difference $t-t^{\prime}$ provides the excitation spectra of the system (it is a very general textbook result; see, for example, [19]). In order to reduce the complexity of the system, we assume the following quasiparticle approximation:

$$
\begin{aligned}
G^{\lessgtr}= & -i h^{\lessgtr}(\mathbf{R}, \mathbf{p}, T, \omega) \mathbb{I}^{+} \delta(\omega-\mathfrak{E}(\mathbf{R}, \mathbf{p}, T)) \\
& -i h^{\gtrless}(\mathbf{R},-\mathbf{p}, T,-\omega) \mathbb{I}^{-} \delta(\omega+\mathfrak{E}(\mathbf{R},-\mathbf{p}, T)) .
\end{aligned}
$$

Here, $h$ is the new unknown of the problem, the superscripts $\lessgtr$ indicate the less- and greater-than Green functions [19], and

$$
\mathbb{I}^{+}=\left(\begin{array}{ll}
1 & 0 \\
0 & 0
\end{array}\right) ; \quad \mathbb{I}^{-}=\left(\begin{array}{cc}
0 & 0 \\
0 & -1
\end{array}\right) .
$$

Equation (15) accounts for two approximations. First, we assume that the solution is constituted by the sum of two long-life excitations with energy $\pm \mathfrak{E}$. This symmetry is a general property of the solution and arises because not all fields that compose the original density matrix given in Eq. (2) are independent. In fact, by using the definition of the Wigner-Weyl transform, it is easy to show that the following property of the function $h$ holds true [6]:

$$
h^{\gtrless}(\mathbf{R},-\mathbf{p}, T,-\omega)=h^{\lessgtr}(\mathbf{R}, \mathbf{p}, T, \omega) .
$$

The second approximation contained in Eq. (15) is that $G$ is assumed to be always a diagonal matrix. This is justified in the limit $\hbar \rightarrow 0$. When Eq. (12) is written explicitly, it is possible to verify that the nondiagonal terms of $G$ vanish at the leading order in $\hbar$. This is analogous to the situation encountered in similar problems describing particle evolution in semiconductor or pseudospin systems [16,18]. With Eq. (16), Eq. (15) simplifies to

$$
\begin{aligned}
G^{\lessgtr}= & -i f \lessgtr(\mathbf{R}, \mathbf{p}, T)\left[\mathbb{I}^{+} \delta(\omega-\mathfrak{E}(\mathbf{R}, \mathbf{p}, T))\right. \\
& \left.+\mathbb{I}^{-} \delta(\omega+\mathfrak{E}(\mathbf{R},-\mathbf{p}, T))\right],
\end{aligned}
$$

where $f \lessgtr(\mathbf{R}, \mathbf{p}, T)=h \lessgtr(\mathbf{R}, \mathbf{p}, T, \omega)$. In particular,

$$
i \int_{\mathbb{R}^{+}} \operatorname{Tr}\left\{G^{\lessgtr}\right\} d \omega=f^{\lessgtr}(\mathbf{R}, \mathbf{p}, T),
$$

where $\operatorname{Tr}$ denotes the trace. Even though the quasiparticle ansatz of Eq. (15) is used, Eq. (12) remains extremely complex to solve. The $\hbar$ expansion provides, systematically, a hierarchy of approximated models. We derive the leading term of the ballistic transport defined by the commutator $[\mathcal{K}, G]_{\star}$. By using Eqs. (8) and (9), after straightforward calculations, Eq. (17) combined with Eq. (12) gives (a similar expression can be obtained for $f^{>}$)

$$
\begin{aligned}
& \frac{\partial f^{<}}{\partial T}+\nabla_{\mathbf{r}} \mathfrak{E} \cdot \nabla_{\mathbf{p}} f^{<}-\left(\mathbf{V}_{c}+\nabla_{\mathbf{p}} \mathfrak{E}\right) \cdot \nabla_{\mathbf{p}} f^{<} \\
& =\frac{1}{\hbar} \int_{\mathbb{R}^{+}} \operatorname{Tr}\{\mathfrak{O}\} d \omega,
\end{aligned}
$$

where $\mathfrak{O}$ denotes the less-than expression of $\mathcal{W}^{-1}\left\{\widehat{\Theta}\left\langle\sigma_{z} \mathfrak{S} \widehat{\Theta}^{-1} \widehat{G}\right\rangle-\left\langle\widehat{G} \widehat{\Theta} \sigma_{z} \mathfrak{S}\right\rangle \widehat{\Theta}^{-1}\right\}$. The term $\mathfrak{O}$ includes all of the particle collision processes. Generally speaking, the particle scattering can be classified in three different type of processes: collisions among noncondensed particles, collisions involving condensed and noncondensed particles, and collisions within the condensate. Since the main interest of this work is to reproduce the processes through which the condensate is formed, we limit ourselves to consider the first two types of collisions. A rich variety of models and studies have established a quite clear understanding of the particle collision processes that occur between a low-density gas and a condensate. In particular, a great effort has been directed to model cold heavy atoms or short-life systems such as polaritons (see, for example, [20]). The analyses reveal that when the conditions for the Bose-Einstein condensation are 
satisfied, namely, when the de Broglie wavelength of a lowtemperature gas is of the same order as the mean interparticle distance, long-range particle-particle correlations should be considered. Under this condition, the standard many-particle expansion of the original Hamiltonian does not apply.

The first theoretical results concerning the many-body ground state for weakly interacting bosons were given by Bogoliubov. The main achievement of his approach was to provide the correct energy dispersion of the elementary gapless excitation of the system. A powerful and sophisticated instrument for the analysis of the many-body ground and excited states is provided by the Baliev theory [3] (see, for example, [21,22] for a clear review of the main results of the theory). Based on this theory, various authors extended the results obtained by Bogoliubov. When all of the secondorder interactions are considered (Hartree-Fock-Bogoliubov approximation), a gap in the excitation energy is introduced. As shown by Griffin, this inconsistency can be avoided by neglecting some nondiagonal terms appearing in the expansion of the self-energy (gapless approximation: the procedure is explained in detail in Ref. [10]). We include in our model the main Hartree-Fock-Bogoliubov scattering processes in the gapless approximation. The related Feynman diagrams can be found, for example, in Ref. [6]. They describe binary collisions between noncondensed particles [Eqs. (B1) and (B2) below] and the scattering processes where only one condensed particle is involved [Eqs. (B3)-(B8) below]. As

an example, the second type of processes includes transitions where one particle is injected in the condensate after collision with another noncondensed particle, and where an atom is expelled from the condensate by collision with the surrounding noncondensed clouds. We do not consider processes where two or more particles in the condensed phase scatter together. For low-density systems, these phenomena are expected to be negligible. They are considered, for example, in Ref. [23].

By performing semiclassical expansion, it is possible to show that the leading term in the collision kernel of Eq. (18) can be written in the standard form,

$$
\int_{\mathbb{R}^{+}} \operatorname{Tr}\{\mathfrak{O}\} d \omega=\int \operatorname{Tr}\left\{\boldsymbol{\Sigma}^{>}\left\langle\mathcal{G}^{<}\right\rangle-\boldsymbol{\Sigma}^{<}\left\langle\mathcal{G}^{>}\right\rangle\right\} d \omega,
$$

where $\Sigma$ is the sum of all of the terms that appear in Eqs. (B1)-(B8). The main idea is that at the zeroth order, the Moyal product reduces to the simple matrix product. The cyclic permutation of the symbols in the curly bracket can be used to eliminate the $\Theta$ matrix.

The calculations proceed straightforwardly. However, since various scattering processes are included, the final result is quite cumbersome. Details of the calculations are given in Appendix B. The equation of motion for the noncondensed phase becomes

$$
\frac{\partial f^{<}}{\partial T}-\nabla_{\mathbf{r}} \mathfrak{E} \cdot \nabla_{\mathbf{p}} f^{<}+\left(\mathbf{V}_{c}+\nabla_{\mathbf{p}} \mathfrak{E}\right) \cdot \nabla_{\mathbf{p}} f^{<}=\mathcal{W}_{H F}+\mathcal{W}_{c n},
$$

where

$$
\begin{gathered}
\mathcal{W}_{H F}=\frac{2 g^{2}}{(2 \pi)^{5}} \int \prod_{j} d \mathbf{p}_{j} \delta\left(\mathfrak{E}(\mathbf{p})+\mathfrak{E}\left(\mathbf{p}_{1}\right)-\mathfrak{E}\left(\mathbf{p}_{2}\right)-\mathfrak{E}\left(\mathbf{p}_{3}\right)\right) \delta\left(\mathbf{p}+\mathbf{p}_{1}-\mathbf{p}_{2}-\mathbf{p}_{3}\right) \\
\times\left[f^{>}(\mathbf{p}) f^{>}\left(\mathbf{p}_{1}\right) f^{<}\left(\mathbf{p}_{2}\right) f^{<}\left(\mathbf{p}_{3}\right)-f^{<}(\mathbf{p}) f^{<}\left(\mathbf{p}_{1}\right) f^{>}\left(\mathbf{p}_{2}\right) f^{>}\left(\mathbf{p}_{3}\right)\right] \mathbf{T}_{H F}, \\
\mathcal{W}_{c n}=\frac{4 g^{2} n_{c}}{(2 \pi)^{2}} \int \prod_{j} d \mathbf{p}_{j} \delta\left(\mathfrak{E}(\mathbf{p})+\mathfrak{E}\left(\mathbf{p}_{1}\right)-\mathfrak{E}\left(\mathbf{p}_{2}\right)\right) \delta\left(\mathbf{p}+\mathbf{p}_{1}-\mathbf{p}_{2}\right)\left[f^{>}(\mathbf{p}) f^{>}\left(\mathbf{p}_{1}\right) f^{<}\left(\mathbf{p}_{2}\right)-f^{<}(\mathbf{p}) f^{<}\left(\mathbf{p}_{1}\right) f^{>}\left(\mathbf{p}_{2}\right)\right] \mathbf{T}_{c n} \\
+\frac{2 g^{2} n_{c}}{(2 \pi)^{2}} \int \prod_{j} d \mathbf{p}_{j} \delta\left(\mathfrak{E}(\mathbf{p})-\mathfrak{E}\left(\mathbf{p}_{1}\right)-\mathfrak{E}\left(\mathbf{p}_{2}\right)\right) \delta\left(\mathbf{p}-\mathbf{p}_{1}-\mathbf{p}_{2}\right)\left[f^{>}(\mathbf{p}) f^{<}\left(\mathbf{p}_{1}\right) f^{<}\left(\mathbf{p}_{2}\right)-f^{<}(\mathbf{p}) f^{>}\left(\mathbf{p}_{1}\right) f^{>}\left(\mathbf{p}_{2}\right)\right] \mathbf{T}_{c n} .
\end{gathered}
$$

The explicit form of the scattering matrices $\mathrm{T}_{H F}$ and $\mathrm{T}_{c n}$ is given in Eqs. (B9) and (B10) and $f^{>}=1+f^{<}$.

\section{CONDENSATE PHASE: GROSS-PITAEVSKII EQUATION}

The condensate atoms are described by the order parameter $\Phi$. Particularly relevant is the norm $n_{c}$ that accounts for the number of particles in the Bose-Einstein state [10]. The evolution equation of $\Phi$ is the so-called Gross-Pitaevskii equation and is a firmly established instrument for the investigation of the ground-state and dynamical excitations of a weakly interacting Bose gas. It describes the motion of the condensate in the presence of the Hartree mean field produced by the other atoms in the condensate. At higher temperatures, and in particular during the cooling process, the interaction with the atoms that are thermally excited out of the condensate cannot be discarded. Zaremba and co-workers showed that the Bolzmann-like approach derived in Sec. III is compatible with the Gross-Pitaevskii (GP) equation, provided that a non-Hermitian term is inserted in the equation for the order parameter [24,25,27]. The evolution equation for the condensate is

$$
\begin{aligned}
i \hbar \frac{\partial \Phi(\mathbf{r}, t)}{\partial t}= & {\left[-\frac{\hbar^{2} \Delta_{\mathbf{r}}}{2 m}+U(\mathbf{r})+g|\Phi(\mathbf{r}, t)|^{2}+2 g n_{c}(\mathbf{r}, t)\right.} \\
& \left.-\frac{i}{n_{c}} \int \mathcal{W}_{c n}(\mathbf{p}) d \mathbf{p}\right] \Phi(\mathbf{r}, t) .
\end{aligned}
$$

The link between the condensed and noncondensed atoms is expressed by the term $\mathcal{W}_{c n}$. It can be easily understood 
in terms of a balance of particles. Since the total number of particles is conserved, a source-sink term that gives the number of particles entering or leaving the condensate is present [26]. We now make a few remarks on the effect of the Hartree-Fock (HF) potential of the noncondensed cloud on the solution of the coupled Boltzmann-GP equations. In the case of a uniform system, the chemical potential of the condensate (obtained by the standard substitution $i \hbar \frac{\partial \Phi(\mathbf{r}, t)}{\partial t} \rightarrow$ $\mu \Phi)$ equals $\mu=U+g n_{c}+2 g n$. Sometime this expression is applied also to nonuniform systems, and in this case is known as the Thomas-Fermi approximation (see, i.e., [6]). The quasiparticle dispersion relation simplifies

$$
\mathfrak{E}=\sqrt{\left[\frac{\mathbf{p}^{2}}{2 m}+\frac{1}{2} m \mathbf{V}_{c}^{2}(\mathbf{r}, t)+g n_{c}(\mathbf{r}, t)\right]^{2}-g^{2} n_{c}^{2}} .
$$

This expression shows that the HF contribution of the noncondensed cloud does not appear explicitly in the expression of the quasiparticle energy. When the Thomas-Fermi approximation is no longer valid, this term modifies the condensate density $n_{c}$ and, indirectly, the energy of the noncondensed particle gas.

\section{A. Numerical applications: Evaporation dynamics}

Equations (20) and (23) describe the interaction between condensed and noncondensed particles. The numerical resolution of this system proceeds with several difficulties. The main reasons are the presence of nonlinearity in the collision kernel and the divergence of the distribution function at $\mathbf{p}=0$. The latter is intrinsically related to the Bose-Einstein-condensation mechanism. The scattering coefficients are strongly nonlinear functions of the local condensate density. The interactions among dressed particles are considerably more complicated than the simple $s$-wave hard-sphere scattering that characterizes the bare positronium. The numerical implementation of the dynamics of a boson gas with bare interactions was already studied [9]. The validity of the model was restricted to the description of the interaction between a warm gas and condensate. In fact, the use of the bare bosons instead of the dressed particles is correct only for large momenta. The study of the condensation processes should require a careful modeling of the dynamical processes that take place in proximity of the condensate energy. As shown in Eq. (14), in our approach, the Bogoliubov excitation spectrum is considered. The dispersion relation of the dressed particles is nonparabolic around $\mathbf{p}=0$, where it becomes linear (gapless phononlike behaviors).

We provide a few details concerning the numerical implementation of the evolution equations. The use of the Bogoliubov spectrum has the advantage that when the condensate density changes, the spectrum of the noncondensed particles is consistently modified. In this case, an energy mesh consisting of equally spaced points is a natural choice. This contrasts with the approach described in Ref. [28] where two discretization meshes are required (a fine and a coarse grid, respectively, for the condensed and noncondensed band is applied) and, in Ref. [29], where a suitable energy transformation is considered. The explicit form of the collision integrals used in the numerical implementation is given in Appendix $\mathrm{C}$ and they are evaluated by the simple trapezoidal rule. The advantage of this choice is that the energy conservation is numerically exact. A fourth-order Runke-Kutta method is used to propagate the solution in time.

We apply our system of equations to the study of the condensation dynamics of a positronium gas. The mass of the atoms is twice the mass of the electron and the bare positronium scattering length is equal to $0.44 \mathrm{~nm}$ [30] (these are the only two parameters required for the calculation of the scattering collision kernels). We consider the simple case of a uniform gas. Despite the drastic reduction of complexity, the nonlinearly coupled gas-condensate dynamics is far from trivial. Since an isolated system conserves the total energy, if we limit ourselves to consider an infinite volume filled with hot bosons, there is no physical mechanism capable of cooling down the gas until the condensation temperature is reached. We propose a simple model that reproduces the particles' cooling by evaporation. We assume that the gas is confined in a box sufficiently large so that all of the spatial variations of the gas and the condensate may be neglected. The confining box is modeled by a potential square well with depth $\Delta_{b}$. The particles with kinetic energy larger than $\Delta_{b}$ can escape the box. Since we assume that the density is uniform inside the confining domain, the parameter $\Delta_{b}$ constitutes a cutoff for the gas distribution $f$. The numerical simulations show that the condensation can be efficiently achieved by using the strongly out-of-equilibrium dynamics induced by the evaporation. At $t=0$, we assume that a thermal distribution of gas is present inside the box and no particles are in the condensate. We make a cutoff in the energy distribution at $\Delta_{b}$ and we let the system evolve. The basic idea of the evaporation cooling process is that after each collision, the outgoing particles with high energy are eliminated. This modifies the standard Boltzmann shape of the energy probability density. The redistribution of the energy among the particles promotes the occupation of low-energy states. The clear disadvantage of this procedure is that a considerable number of particles is lost.

In Fig. 1, the time evolution of the condensed density is depicted. In order to illustrate the results, we consider two different initial temperatures, $T=30 \mathrm{~K}$ (continuous blue curve) and $T=50 \mathrm{~K}$ (dash-dotted red curve) and two values of the cutoff $\Delta_{b}: 0.5 \mathrm{meV}$ (left panel) and $0.3 \mathrm{meV}$ (right panel). The simulations show that the condensation process is achieved in nearly 100 ps. This time interval is quite short and could be interesting for short-lifetime atoms such as positronium. Figure 1 (right panel) shows that decreasing the parameter $\Delta_{b}$ makes the production of condensate more efficient in terms of the fraction of the original number of particles. However, by reducing the value of the cutoff, the number of particles lost in the process increases. If instead of relative units, we consider the final density of particles, then the cutoff at $0.3 \mathrm{meV}$ leads to a condensate with density equal to $1.2 \mathrm{~nm}^{-3}$ (initial temperature $50 \mathrm{~K}$ ), while the cutoff at $0.5 \mathrm{meV}$ (for the same initial condition for the particle distribution) leads to density around $1.6 \mathrm{~nm}^{-3}$. As a consequence, a compromise should be found between having an efficient production of cold particles and the necessity to preserve a sufficiently high number of atoms. This is illustrated in Fig. 2, where the evolution of the total density of particles is depicted (continuous line). The simulations show that nearly 

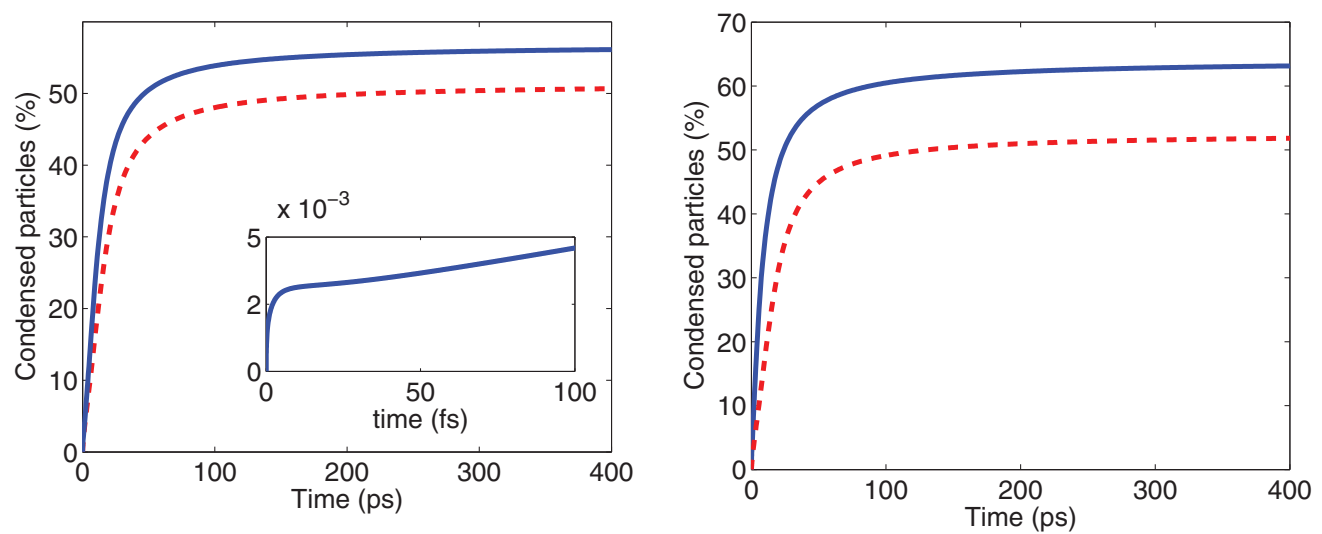

FIG. 1. (Color online) Time evolution of the density of condensed particles (\% of the initial density of particles) for different initial temperatures of the gas: $T=30 \mathrm{~K}$ (continuous blue curve) and $T=50 \mathrm{~K}$ (dash-dotted red curve). Energy cutoff: $0.5 \mathrm{meV}$ (left panel) and $0.3 \mathrm{meV}$ (right panel). The inset in the left panel displays the initial fast dynamical evolution of the density corresponding to $T=30 \mathrm{~K}$.

half of the particles are expelled from the system (we remark that when we use a smaller energy cutoff, the initial density of particles is consequently reduced). The comparison with the condensate density (dashed line) shows that the condensate production is quite efficient and more than $80 \%$ of the particles that remain in the system belongs to the condensate (we remark that in Fig. 1, we plot the ratio between the condensed density and the initial density of the gas).

In Fig. 1 (inset), we emphasized the extremely fast initial production of the condensate. This behavior is due to the considerable change of the scattering coefficients when the condensate density is no longer zero. This point is more clearly illustrated in Fig. 3, where we highlight the importance of the corrections to the two-particle scattering rate induced by the quasiparticle description of the noncondensed atoms. The presence of the condensate alters considerably the simple hard-sphere scattering process that characterizes the bare positronium. In our model, the scattering rates are expressed by the functions $\mathrm{T}_{n c}$ and $\mathrm{T}_{n n}$ of Eqs. (21) and (22). The dressed interaction effects can be easily removed by setting $\mathrm{T}_{n c}=\mathrm{T}_{n n}=1$. The result is depicted in Fig. 3, where we compare the evolution of the condensate density in the absence of any correlation effect (dotted red curve). The simulation shows clearly that for a low-mass atom such as positronium, the use of the correct Bogoliubov representation of the atomic gas leads to a significant modification of the condensate evolution. Most of the systems considered for BEC are made of heavy particles and this aspect is usually neglected.

In Fig. 4, we depict the distribution function of the gas multiplied by the density of states for different times. The energy cutoff is clearly visible. During the evolution, the energy gap at $\Delta_{b}$ decreases and nearly vanishes at 500 ps. We remark that when a cutoff in the particle distribution is imposed, the stationary state reached by the system is no longer a Bose-Einstein distribution. For comparison, in Fig. 4 (right panel), we compare the final particle distribution with a Bose-Einstein distribution with zero chemical potential and temperature of $2 \mathrm{~K}$ (dashed green line). Fitting the particle distribution with the equilibrium distribution provides an estimate of the temperature reached by the gas (although, strictly speaking, in this nonequilibrium situation, the temperature is not a well-defined quantity).
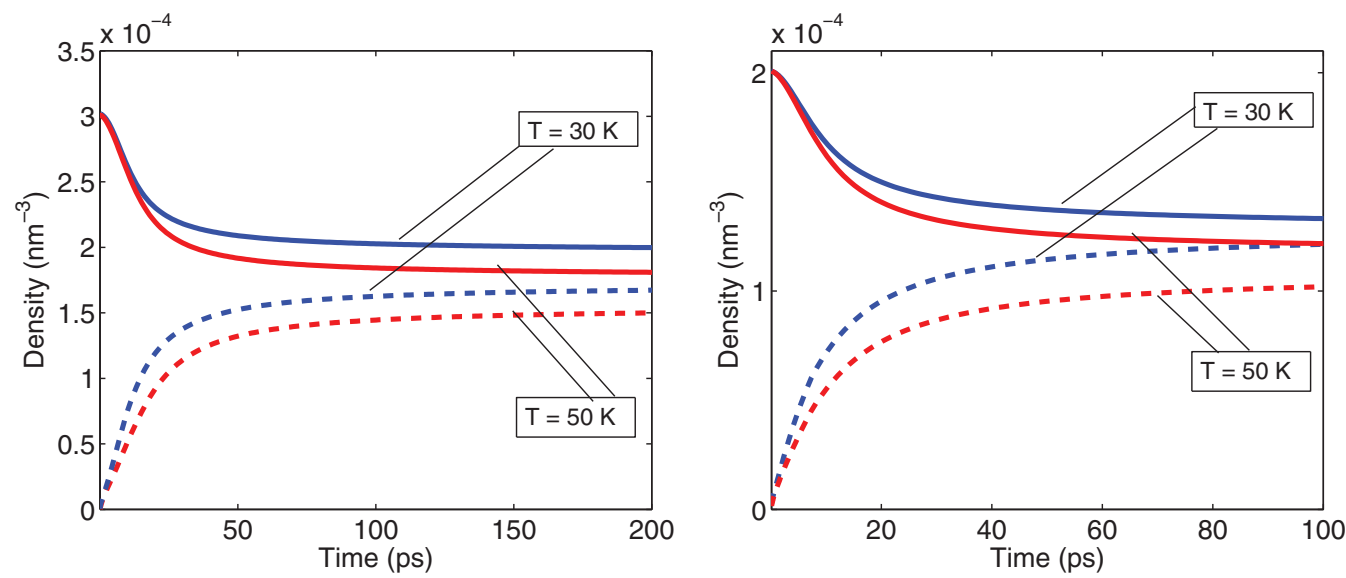

FIG. 2. (Color online) Evolution of the total density of particles (noncondensed plus condensed particles) (continuous curves) and condensed density (dashed curves) for different initial temperatures: $T=30 \mathrm{~K}$ (blue curves) and $T=50 \mathrm{~K}$ (red curves). Energy cutoff: $0.5 \mathrm{meV}$ (left panel) and $0.3 \mathrm{meV}$ (right panel). 


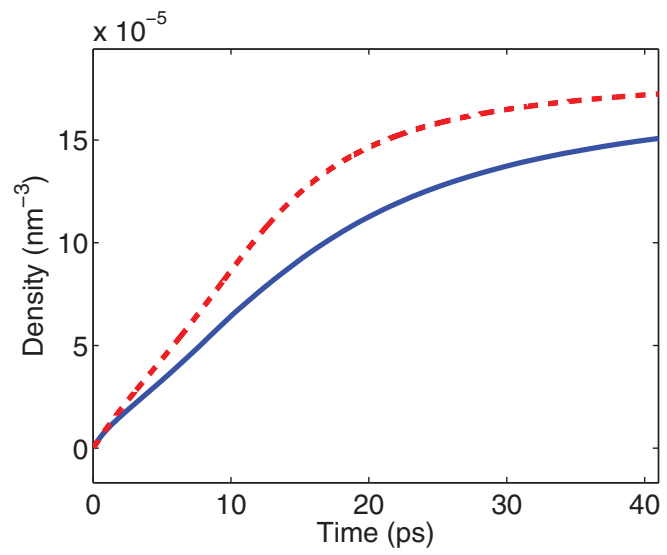

FIG. 3. (Color online) Modification of the condensate growth induced by the corrections on the scattering integrals that came from the correlation between condensed and noncondensed particles (dressed interaction). The dotted red curve is obtained by considering only the bare interaction (hard-sphere scattering) and the continuous blue curve is obtained by taking into account the full model. In the simulations, we used the same parameters as in Fig. 2 (left panel) and $T=30 \mathrm{~K}$.

\section{COMPARISON WITH OTHER APPROACHES}

Various theoretical and numerical approaches have been developed for the reproduction of the condensation dynamics for cold atomic gases or collective excitations such as excitons and polaritons. The first quantitative descriptions of the condensate growth have been derived by Snoke [31], Semikoz [32], and Tassone [11]. These approaches were based on the classical Boltzmann equation. Although we derived our kinetic model in a full quantum context, the final equations of motion maintain the mathematical structure of the former theories and can be directly compared. Major advances on the theory of the condensate growth have been performed by the groups of Gardiner [33] and Zaremba [29]. Their numerical predictions on the sodium condensation dynamics have been compared with experiments. Although the final set of equations that we used to simulate the positronium evolution are quite

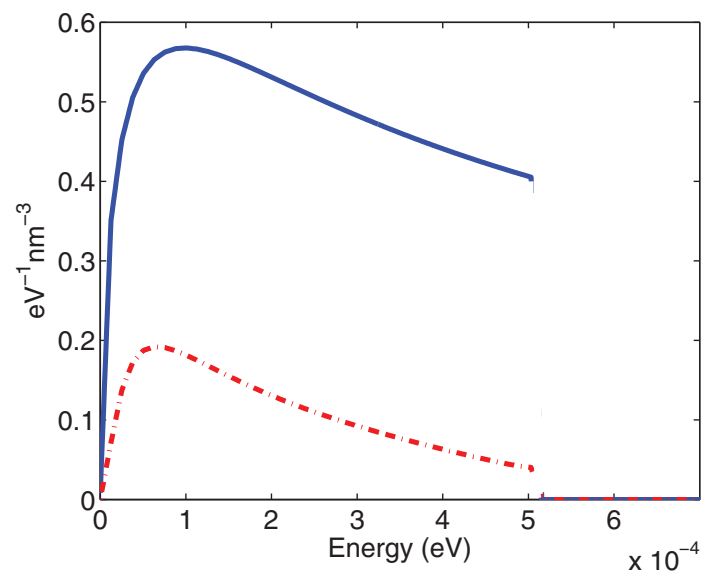

similar to the analogous set used for sodium, some important differences should be noticed. The main difference between our approach and the model described in Ref. [33] concerns the description of the condensate. In our formulation, the condensate is treated from the start as a macroscopic quantum state and the quantum-mechanical nature of the interaction with the surrounding thermal cloud is addressed explicitly. As a result, important qualitative modifications to the collision integrals are obtained. In particular, the scattering rate that describes the scattering between two particles in the cloud has a quite complicated form and differs significantly from the term that describes the collision between one particle in the condensate and one particle in the noncondensed cloud. This distinction is not taken into account in Ref. [33], while in the model given in Ref. [29], it is included only by the modification of the total collision cross section.

In Gardiner's approach [33], in order to take into account the self-consistent mean field of the condensate and its influence on the evolution of the system with sufficient accuracy, the particles in the cloud are divided into two subgroups: those belonging to the condensate band and those belonging to the noncondensate band. The condensed band groups together the states whose energy is modified by the mean field of the condensate. On the contrary, above a certain value of the energy (which is the lower bound of the noncondensed band), the particle spectrum is considered as constant. Since in this approach the condensed band represents the ensemble of the quasiparticle states, the calculations of the density of states and the collision integrals should be coherently performed by using the Bogoliubov excitation spectrum instead of the bare spectrum. The use of the bare-particle picture is necessary if the corrections to the total energy of the particle given by the term $g^{2} n_{c}^{2}$ are of the same order as the kinetic energy of the free particle. Since the bare interaction $g$ is proportional to the inverse of the particle mass, these corrections are important only for light particles. As showed in Fig. 3, for light mass particles such as the positronium, such a correction affects the growth rate and the final value of the number of condensed particles. Furthermore, the use of the quasiparticle spectrum has the advantage that the modification of the energy levels induced

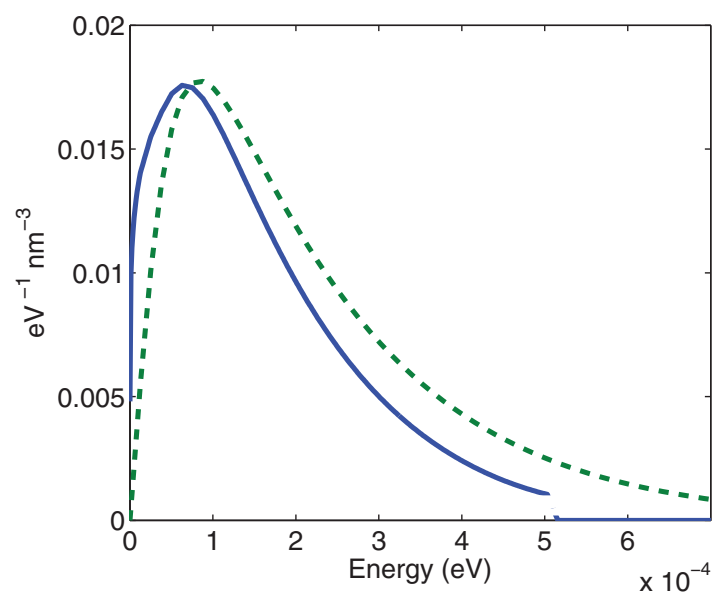

FIG. 4. (Color online) Noncondensed distribution function multiplied by the density of states for different times. Left panel: $t=50 \mathrm{fs}$ (blue curve) and $t=30 \mathrm{ps}$ (red curve). Right panel: same case for $t=500 \mathrm{ps}$. For the sake of comparison, the dashed green curve inside the right panel depicts a Bose-Einstein distribution at $2 \mathrm{~K}$. The initial distribution corresponds to the initial temperature of $50 \mathrm{~K}$. 
by the presence of the condensate is automatically taken into account [see the definition given in Eq. (14)] and there is no need to distinguish between particles with energy close to or far from the chemical potential of the condensate.

Other models where the Bogoliubov dressed particle approach is used were derived by Kirkpatrick [14] and Griffin [6]. The main difference with our approach is essentially technical and concerns the implementation of the Bogoliubov transformation. In Ref. [14], a local Bogoliubov transformation is defined. The dressed field operators are expressed in terms of the free bare-particle operators. They assume that the expansion coefficients change in space and adapt themselves to the condensate density present at the same position. This is equivalent to assuming that the system is characterized by a macroscopic scale length and that the main physical quantities change smoothly in space. This relies on a series of approximations that involve classical, large-scattering length and low-density limits. All of these assumptions make it difficult to estimate the exact range of validity of the approximations. Furthermore, they do not indicate a strategy that could be applied to overcome these approximations and obtain more accurate models. A similar procedure is used in Ref. [15], where the positive and the negative energy spectrum of the excitations is diagonalized by using the same kind of approximations. In our approach, we derive the Bogoliubov transformation in a full quantum regime, and no approximations are required for the definition of the Wigner distribution in the Bogoliubov basis. Furthermore, we provide a systematic procedure (in terms of the $\hbar$ expansion) through which higher-order corrections to the scattering kernels can be included.

As a final remark, we discuss some features of the behavior of the positronium density function when the condensate starts to form. The dynamical evolution of a Bose gas at the supercritical regime has been deeply investigated both from the theoretical and the numerical point of view. The early stage of the formation of the condensate is a two-step process where the transition between the two regimes take place at the critical time $t_{c}$. During the first stage, the distribution function is characterized by the formation of a divergence in correspondence to $p=0$. Asymptotically, the pole behaves like $E^{-7 / 6}$ [34] (this was also somehow reproduced by the numerical simulations performed in Ref. [32]). After the critical time, higher-energy levels start to be populated and a fast increase in the number of particles in the condensate is observed. On the basis of some classical considerations, the critical time $t_{c}$ can be estimated by the mean collision time $t_{c}=\frac{m}{8 \pi a^{2} n \sqrt{2 k_{B} T}}$, where $n$ is the noncondensate density and $T$ is the temperature [35]. For the positronium, the typical values used in our simulations lead to the estimation of $t_{c} \simeq 10^{-1} \mathrm{ps}$. In our simulations, we observe that the extremely fast growth of the condensate during the early stage of the dynamics (see Fig. 1) prevents the formation of the strong divergence in the distribution function. On the contrary, this is observed for heavy particles such as sodium, where $t_{c}$ is of the order of $10 \mathrm{~ms}$, while the condensation takes nearly $0.1 \mathrm{~s}$ (see Fig. 5) [36].

As a benchmark of our theory, we calculate the growth of a condensate made of ${ }^{23} \mathrm{Na}$ atoms trapped in a harmonic potential. The theoretical study of this process and the comparison with experiments were already addressed by other authors [29,36]. In the experiments described in Ref. [37], a cloud of sodium atoms is cooled to just above the transition temperature. The atoms are confined in an axially symmetric trap with harmonic frequencies equal to 18.0 and $82.3 \mathrm{~Hz}$ along and perpendicular to the symmetry axis, respectively. In order to simplify the geometry of the system, we approximate the trap with a symmetric confining potential with an averaged frequency $\omega$ equal to $49.6 \mathrm{~Hz}$. The condensation dynamics is triggered by applying a radio-frequency signal that removes the high-energy tail of the distribution. We note that due to the heavy mass of sodium, the corrections to the scattering rate between two atoms induced by the presence of the condensate are negligible and the binary collisions are well represented by the $s$-wave scattering (the scattering length is equal to $2.75 \mathrm{~nm}$ ). In this case, the atomic energy is given by the usual parabolic law, $E=\frac{p^{2}}{2 m}+2 \mu_{c}-U$. According to $[29,33]$, we include the mean-field effects via a Thomas-Fermi approximation. In this way, the chemical potential of the condensate in the harmonic potential becomes

$$
\mu_{c}=\frac{\hbar \omega}{2}\left(15 N_{c} a \sqrt{\frac{m \omega}{\hbar}}\right)^{2 / 5} .
$$

Before the atoms are expelled from the trap, the gas temperature $T_{i}$ is equal to $1119 \mathrm{nK}$ and the distribution of the gas is a Bose-Einstein function with chemical potential $\mu$ equal to $-100 \hbar \omega$. We then cut the distribution at the energy, $E_{c u t}=1.4 k_{B} T_{i}$.

The results of our calculations are shown in Fig. 5. In Figs. 5(a)-5(c), we depict the evolution of the gas toward the final equilibrium state. In agreement with the numerical results of Ref. [28], the thermalization process takes place in around $0.6 \mathrm{~s}$. The growth of the number of particles in the condensate is depicted in Fig. 5(d). After an initial exponential growth of the condensate, the condensate needs around $0.1 \mathrm{~s}$ to incorporate $10^{6}$ atoms. The initial number of particles in the condensate (in our simulation, we set this number equal to 100) does not affect the growth rate of the condensed atoms.

Our calculations are qualitatively in agreement with the condensate evolution obtained in Refs. [29] and [28]. The main difference concerns the final number of particles in the condensate (our computed value is nearly double that obtained by the other authors) and the time where the condensation process starts to slow down (in our simulation, it is around $0.1 \mathrm{~s}$; in [28], it is $0.05 \mathrm{~s}$; and in [29], it is $0.01 \mathrm{~s}$ ). We believe that the source of the discrepancy is mainly due to the fact that in our simulation, we do not use the correct density of states of the trap. Since the principal aim of our approach was to describe the modification of the particle energy and interaction in the presence of a condensate, in our model we discarded the modification of the particle energy induced by the confining potential and we considered the density of state of an isotropic three-dimensional (3D) system. However, the three-dimensional trap used in the experiments possesses a relatively small number of isolated levels and the particle energies rapidly approach the continuum. For this reason, discarding the correct distribution of the low-energy bound states does not have a dramatic influence on the final results and our model is able to reproduce the correct order of magnitude of the condensation time and number of condensed particles. 

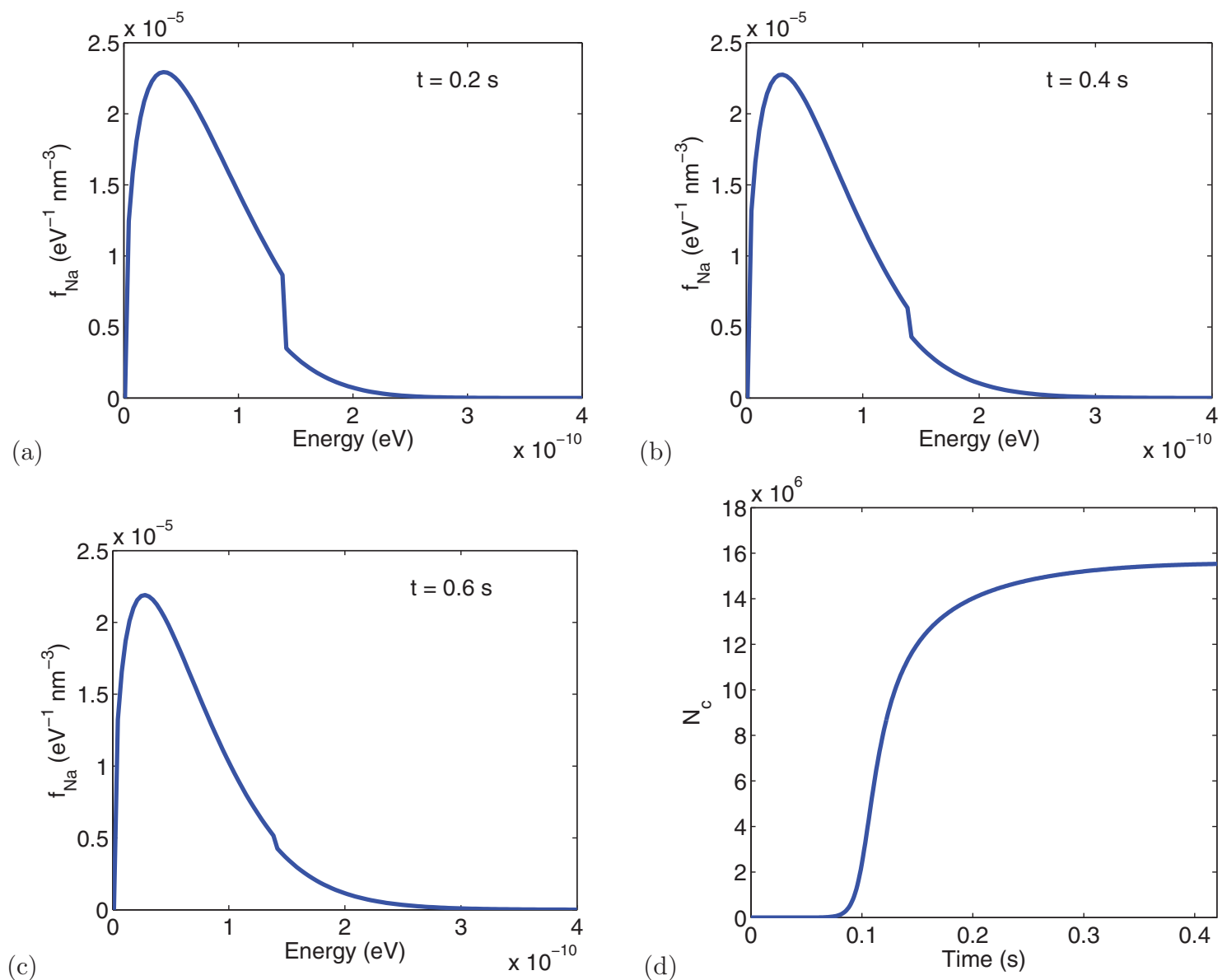

(b)

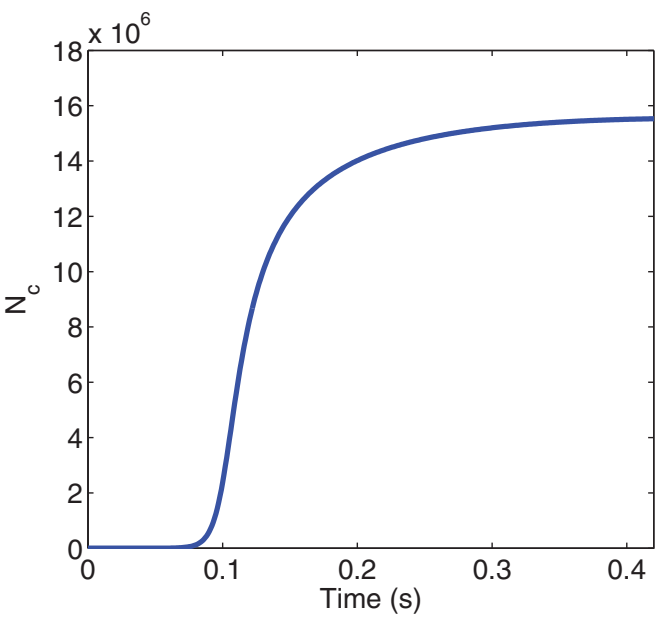

FIG. 5. (Color online) Evolution of the density of the ${ }^{13} \mathrm{Na}$ cloud during the condensation process. The snapshots correspond to the time (a) $0.2 \mathrm{~s}$, (b) $0.4 \mathrm{~s}$, and (c) $0.6 \mathrm{~s}$. (d) Evolution of the number of particles in the sodium condensate.

\section{CONCLUSIONS}

The study of the condensation dynamics was performed by combining the semiclassical Boltzmann dynamics of noncondensed particles with the Gross-Pitaevskii equation for the description of the condensate state. We modeled the evaporation process by inserting a cutoff in the particle distribution function. The system shows a strong out-of-equilibrium dynamics and fast condensation time (around one-hundred picoseconds). Our results suggest the possibility of using this technique for the study of the condensation process of short-lifetime atoms such as positronium.

\section{ACKNOWLEDGMENT}

This work was partially funded by the Agence Nationale de la Recherche (Contract No. ANR-10-BLAN-0420).

\section{APPENDIX A: BOGOLIUBOV TRANSFORMATION IN THE WEYL FORMALISM}

We show the connection of the basis rotation given in Eq. (13) and the Bogoliubov transformation. The wave function related to the basis rotation generated by $\Theta$ is given by

$$
\begin{aligned}
\Psi^{\prime}(\mathbf{r}, t)= & \frac{1}{(2 \pi \hbar)^{3}} \int \Theta\left(\frac{\mathbf{r}+\mathbf{r}^{\prime}}{2}, \mathbf{p}\right) \Psi\left(\mathbf{r}^{\prime}, t\right) e^{\frac{i}{\hbar}\left(\mathbf{r}-\mathbf{r}^{\prime}\right) \cdot \mathbf{p}} d \mathbf{r}^{\prime} d \mathbf{p} \\
\Psi^{\prime}(\mathbf{r}, t)= & \frac{1}{(2 \pi \hbar)^{3}} \sum_{n} \frac{1}{n !}\left(-\frac{i \hbar}{2}\right)^{n} \\
& \times \int \Theta^{(n)}(\mathbf{r}, \mathbf{p}) \Psi\left(\mathbf{r}^{\prime}, t\right) e^{\frac{i}{\hbar}\left(\mathbf{r}-\mathbf{r}^{\prime}\right) \cdot \mathbf{p}} d \mathbf{r}^{\prime} d \mathbf{p},
\end{aligned}
$$

where $\Theta^{(n)}(\mathbf{r}, \mathbf{p})=\nabla_{\mathbf{p}}^{(n)} \nabla_{\mathbf{r}}^{(n)} \Theta(\mathbf{r}, \mathbf{p})$. In particular, at the leading order, the $\hbar$ expansion of Eq. (A2) is the space-dependent Bogoliubov transformation for the field operators of noncondensed particles,

$$
\begin{aligned}
\left(\begin{array}{c}
\tilde{\psi}^{\prime}(\mathbf{r}, t) \\
\widetilde{\psi}^{\prime \dagger}(\mathbf{r}, t)
\end{array}\right)= & \frac{1}{(2 \pi \hbar)^{3}} \int\left(\begin{array}{c}
\mathfrak{u}(\mathbf{r}, \mathbf{p}, t)-\mathfrak{v}(\mathbf{r}, \mathbf{p}, t) \\
\mathfrak{v}(\mathbf{r}, \mathbf{p}, t)-\mathfrak{u}(\mathbf{r}, \mathbf{p}, t)
\end{array}\right) \\
& \times\left(\begin{array}{c}
\widetilde{\psi}\left(\mathbf{r}^{\prime}, t\right) \\
\widetilde{\psi}^{\dagger}\left(\mathbf{r}^{\prime}, t\right)
\end{array}\right) e^{\frac{i}{\hbar}\left(\mathbf{r}-\mathbf{r}^{\prime}\right) \cdot \mathbf{p}} d \mathbf{r}^{\prime} d \mathbf{p}+o(\hbar),
\end{aligned}
$$

where

$$
\mathfrak{u}(\mathbf{r}, \mathbf{p}, t)=\sqrt{\frac{\mathfrak{e}+\mathfrak{E}}{2 \mathfrak{E}}}, \quad \mathfrak{v}(\mathbf{r}, \mathbf{p}, t)=\sqrt{\frac{\mathfrak{e}-\mathfrak{E}}{2 \mathfrak{E}}} .
$$

The functions $\mathfrak{e}$ and $\mathfrak{E}$ are given by Eqs. (14) and (10), respectively. 


\section{APPENDIX B: EXPLICIT EVALUATION OF THE SCATTERING KERNELS}

The scattering processes that we include in our model are the direct and exchange (Hartree-Fock) binary collisions between noncondensed atoms and collisions between condensed and noncondensed atoms (hereafter, we will refer to the "less-than" component of the self-energy; the "greater-than" expressions are obtained by interchanging the symbol $<$ with $>$, and vice versa). The first type of collisions is expressed by

$$
\begin{gathered}
\Sigma_{H}^{<}(\mathbf{p}, \omega)=\frac{g^{2}}{(2 \pi)^{5}} \int \mathcal{G}^{<}\left(\mathbf{p}_{2}, \omega_{2}\right) \operatorname{tr}\left\{\mathcal{G}^{>}\left(\mathbf{p}_{3}, \omega_{3}\right) \mathcal{G}^{<}\left(\mathbf{p}_{4}, \omega_{4}\right)\right\} \mathcal{D} \\
\Sigma_{F}^{<}(\mathbf{p}, \omega)=\frac{2 g^{2}}{(2 \pi)^{5}} \int \mathcal{G}^{<}\left(\mathbf{p}_{2}, \omega_{2}\right) \mathcal{G}^{>}\left(\mathbf{p}_{3}, \omega_{3}\right) \mathcal{G}^{<}\left(\mathbf{p}_{4}, \omega_{4}\right) \mathcal{D}
\end{gathered}
$$

where

$$
\mathcal{D}=\delta\left(\omega_{1}-\omega_{2}+\omega_{3}-\omega_{4}\right) \delta\left(\mathbf{p}_{1}-\mathbf{p}_{2}+\mathbf{p}_{3}-\mathbf{p}_{4}\right) \prod_{j} d \mathbf{p}_{j} d \omega_{j}
$$

The collisions between condensed and noncondensed atoms are expressed by

$$
\begin{aligned}
& \Sigma_{1}^{<}\left(\mathbf{p}_{1}, \omega_{1}\right)=c \int \mathcal{G}^{<}\left(\mathbf{p}_{2}, \omega_{2}\right) \operatorname{tr}\left\{\mathfrak{C}^{<}\left(\mathbf{p}_{3}, \omega_{3}\right) \mathcal{G}^{<}\left(\mathbf{p}_{4}, \omega_{4}\right)\right\} \mathcal{D}, \\
& \Sigma_{2}^{<}\left(\mathbf{p}_{1}, \omega_{1}\right)=c \int \mathcal{G}^{<}\left(\mathbf{p}_{2}, \omega_{2}\right) \operatorname{tr}\left\{\mathcal{G}^{>}\left(\mathbf{p}_{3}, \omega_{3}\right) \mathfrak{C}\left(\mathbf{p}_{4}, \omega_{4}\right)\right\} \mathcal{D} \\
& \Sigma_{3}^{<}\left(\mathbf{p}_{1}, \omega_{1}\right)=c \int \mathfrak{C}\left(\mathbf{p}_{2}, \omega_{2}\right) \operatorname{tr}\left\{\mathcal{G}^{>}\left(\mathbf{p}_{3}, \omega_{3}\right) \mathcal{G}^{<}\left(\mathbf{p}_{4}, \omega_{4}\right)\right\} \mathcal{D}, \\
& \Sigma_{4}^{<}\left(\mathbf{p}_{1}, \omega_{1}\right)=2 c \int \mathcal{G}^{<}\left(\mathbf{p}_{2}, \omega_{2}\right) \mathcal{G}^{>}\left(\mathbf{p}_{3}, \omega_{3}\right) \mathfrak{C}\left(\mathbf{p}_{4}, \omega_{4}\right) \mathcal{D}, \\
& \Sigma_{5}^{<}\left(\mathbf{p}_{1}, \omega_{1}\right)=2 c \int \mathfrak{C}\left(\mathbf{p}_{2}, \omega_{2}\right) \mathcal{G}^{>}\left(\mathbf{p}_{3}, \omega_{3}\right) \mathcal{G}^{<}\left(\mathbf{p}_{4}, \omega_{4}\right) \mathcal{D}, \\
& \Sigma_{6}^{<}\left(\mathbf{p}_{1}, \omega_{1}\right)=2 c \int \mathcal{G}^{<}\left(\mathbf{p}_{2}, \omega_{2}\right) \mathfrak{C}^{<}\left(\mathbf{p}_{3}, \omega_{3}\right) \mathcal{G}^{<}\left(\mathbf{p}_{4}, \omega_{4}\right) \mathcal{D},
\end{aligned}
$$

where $c=-\frac{g^{2}}{2(2 \pi)^{3}}$. For the derivation of these scattering kernels and the relative Feynman diagrams, we refer to [15]. Following Abrikosov [38], we introduced the condensate Green function in a matrix form,

$$
\mathfrak{C}_{0}(\mathbf{R}, \mathbf{p}, T)=-i \mathcal{W}^{-1}\left(\begin{array}{ll}
\Phi(\mathbf{r}, t) \Phi\left(\mathbf{r}^{\prime}, t^{\prime}\right) & \Phi(\mathbf{r}, t) \Phi\left(\mathbf{r}^{\prime}, t^{\prime}\right) \\
\Phi(\mathbf{r}, t) \bar{\Phi}\left(\mathbf{r}^{\prime}, t^{\prime}\right) & \bar{\Phi}(\mathbf{r}, t) \Phi\left(\mathbf{r}^{\prime}, t^{\prime}\right)
\end{array}\right) .
$$

The simplest approximation is to consider $\Phi$ slowly varying in space and time, so that the Wigner function is concentrated around $\mathbf{p}=0$. In this way,

$$
\mathfrak{C}_{0}(\mathbf{R}, \mathbf{p}, T) \simeq-i n_{c}(\mathbf{R}, T) 2 \pi \delta(\mathbf{p}) \delta(\omega) \mathfrak{J}
$$

where

$$
\mathfrak{J}=\left(\begin{array}{ll}
1 & 1 \\
1 & 1
\end{array}\right)
$$

In the previous expressions, the scattering kernels are expressed in terms of integrals of the Green function over the energy variable $\omega$. The quasiparticle closure hypothesis allows us to recover a closed evolution system for the distribution function $f$. Equations (B1) and (B2), as well as (B3)-(B8), show that all of the scattering terms share the following mathematical form:

$$
\begin{aligned}
I(\mathbf{p}, \omega)= & \int \mathcal{G}_{1}^{a_{1}}\left(\mathbf{p}_{1}, \omega_{1}\right) \mathcal{G}^{a_{2}}\left(\mathbf{p}_{2}, \omega_{2}\right) \mathcal{G}^{a_{3}}\left(\mathbf{p}_{3}, \omega_{3}\right) \mathcal{G}^{a_{4}}\left(\mathbf{p}_{4}, \omega_{4}\right) \\
& \times \delta\left(\omega_{1}+\omega_{2}-\omega_{3}-\omega_{4}\right) \delta\left(\mathbf{p}_{1}+\mathbf{p}_{2}-\mathbf{p}_{3}-\mathbf{p}_{4}\right) \delta\left(\omega-\omega_{1}\right) \delta\left(\mathbf{p}-\mathbf{p}_{1}\right) \prod_{j} d \mathbf{p}_{j} d \omega_{j},
\end{aligned}
$$

where the $a_{i}$ take the values $>$ or $<$. From the closure relationship given in Eq. (15), we have that each Green function can be expressed as (we adopt the notation that the overbar means the logical NOT operator: $\overline{<} \equiv>, \overline{>} \equiv<$ )

$$
\mathcal{G}^{a}(\mathbf{p}, \omega)=f^{a}(\mathbf{p}) \mathcal{M}(\mathbf{p}) \delta(\omega-\mathfrak{E}(\mathbf{p}))+f^{\bar{a}}(-\mathbf{p}) \mathcal{M}^{\perp}(-\mathbf{p}) \delta(\omega+\mathfrak{E}(-\mathbf{p})),
$$

with the exception [according to Eq. (17), the final integral energy integral is taken only over the positive frequency]

$$
\mathcal{G}_{1}^{a}(\mathbf{p}, \omega)=f^{a}(\mathbf{p}) \mathcal{M}(\mathbf{p}) \delta(\omega-\mathfrak{E}(\mathbf{p})) .
$$


We defined

$$
\mathcal{M}=\Theta^{-1} \mathbb{I}^{+} \Theta \sigma_{z}=\frac{1}{2 E}\left(\begin{array}{cc}
E+\mathfrak{e}_{B} & g n_{c} \\
g n_{c} & \mathfrak{e}_{B}-E
\end{array}\right), \quad \mathcal{M}^{\perp}=\mathcal{M}-\sigma_{z} .
$$

We remark that the self-energies are expressed in terms of the Green functions on the unperturbed basis $\langle\mathcal{G}\rangle=\Theta^{-1} \star G \star \Theta \sigma_{z}$. The condensate propagator $\mathfrak{C}_{0}$ can be considered a particular case of $\mathcal{G}^{a}(\mathbf{p}, \omega)$, with $\mathfrak{E}(\mathbf{p})=0, \mathcal{M}=\mathcal{M}^{\perp}=\mathfrak{J}$, and $f^{a}(\mathbf{p})=$ $-i n_{c} \pi \delta(\mathbf{p})$. A long but straightforward calculation shows

$$
\begin{aligned}
I= & \int\left\{f^{a_{1}}(\mathbf{p}) f^{a_{2}}\left(\mathbf{p}_{2}\right) f^{a_{3}}\left(\mathbf{p}_{3}\right) f^{\bar{a}_{4}}\left(\mathbf{p}_{4}\right) \mathcal{M}(\mathbf{p}) \mathcal{M}\left(\mathbf{p}_{2}\right) \mathcal{M}\left(\mathbf{p}_{3}\right) \mathcal{M}^{\perp}\left(\mathbf{p}_{4}\right)\right. \\
& +f^{a_{1}}(\mathbf{p}) f^{a_{4}}\left(\mathbf{p}_{2}\right) f^{a_{3}}\left(\mathbf{p}_{3}\right) f^{\bar{a}_{2}}\left(\mathbf{p}_{4}\right) \mathcal{M}(\mathbf{p}) \mathcal{M} \perp\left(\mathbf{p}_{4}\right) \mathcal{M}\left(\mathbf{p}_{3}\right) \mathcal{M}\left(\mathbf{p}_{2}\right) \\
& \left.+f^{a_{1}}(\mathbf{p}) f^{\bar{a}_{3}}\left(\mathbf{p}_{2}\right) f^{\bar{a}_{2}}\left(\mathbf{p}_{3}\right) f^{\bar{a}_{4}}\left(\mathbf{p}_{4}\right) \mathcal{M}(\mathbf{p}) \mathcal{M}^{\perp}\left(\mathbf{p}_{3}\right) \mathcal{M}^{\perp}\left(\mathbf{p}_{2}\right) \mathcal{M}^{\perp}\left(\mathbf{p}_{4}\right)\right\} \\
& \times \delta\left(\mathfrak{E}(\mathbf{p})-\mathfrak{E}\left(\mathbf{p}_{2}\right)+\mathfrak{E}\left(\mathbf{p}_{3}\right)+\mathfrak{E}\left(\mathbf{p}_{4}\right)\right) \delta\left(\mathbf{p}-\mathbf{p}_{2}+\mathbf{p}_{3}+\mathbf{p}_{4}\right) \prod d \mathbf{p}_{j} d \omega_{j} \\
& +\int\left\{f^{a_{1}}(\mathbf{p}) f^{a_{2}}\left(\mathbf{p}_{2}\right) f^{a_{3}}\left(\mathbf{p}_{3}\right) f^{a_{4}}\left(\mathbf{p}_{3}\right) \mathcal{M}(\mathbf{p}) \mathcal{M}\left(\mathbf{p}_{2}\right) \mathcal{M}\left(\mathbf{p}_{4}\right) \mathcal{M}\left(\mathbf{p}_{3}\right)\right. \\
& +f^{a_{1}}(\mathbf{p}) f^{\bar{a}_{3}}\left(\mathbf{p}_{3}\right) f^{\bar{a}_{2}}\left(\mathbf{p}_{4}\right) f^{a_{4}}\left(\mathbf{p}_{2}\right) \mathcal{M}(\mathbf{p}) \mathcal{M}\left(\mathbf{p}_{4}\right) \mathcal{M} \mathcal{M}^{\perp}\left(\mathbf{p}_{3}\right) \mathcal{M}\left(\mathbf{p}_{2}\right) \\
& \left.+f^{a_{1}}(\mathbf{p}) f^{a_{2}}\left(\mathbf{p}_{2}\right) f^{\bar{a}_{3}}\left(\mathbf{p}_{3}\right) f^{\bar{a}_{4}}\left(\mathbf{p}_{4}\right) \mathcal{M}(\mathbf{p}) \mathcal{M}\left(\mathbf{p}_{2}\right) \mathcal{M}^{\perp}\left(\mathbf{p}_{3}\right) \mathcal{M} \mathcal{M}^{\perp}\left(\mathbf{p}_{4}\right)\right\} \\
& \times \delta\left(\mathfrak{E}(\mathbf{p})-\mathfrak{E}\left(\mathbf{p}_{2}\right)-\mathfrak{E}\left(\mathbf{p}_{3}\right)+\mathfrak{E}\left(\mathbf{p}_{4}\right)\right) \delta\left(\mathbf{p}-\mathbf{p}_{2}-\mathbf{p}_{3}+\mathbf{p}_{4}\right) \prod d \mathbf{p}_{j} d \omega_{j} \\
& +\int f^{a_{1}}(\mathbf{p}) f^{a_{2}}\left(\mathbf{p}_{2}\right) f^{\bar{a}_{3}}\left(\mathbf{p}_{3}\right) f^{a_{4}}\left(\mathbf{p}_{4}\right) \mathcal{M}(\mathbf{p}) \mathcal{M}\left(\mathbf{p}_{2}\right) \mathcal{M}^{\perp}\left(\mathbf{p}_{3}\right) \mathcal{M}\left(\mathbf{p}_{4}\right) \\
& \times \delta\left(\mathfrak{E}(\mathbf{p})-\mathfrak{E}\left(\mathbf{p}_{2}\right)-\mathfrak{E}\left(\mathbf{p}_{3}\right)-\mathfrak{E}\left(\mathbf{p}_{4}\right)\right) \delta\left(\mathbf{p}-\mathbf{p}_{2}-\mathbf{p}_{3}-\mathbf{p}_{4}\right) \prod d \mathbf{p}_{j} d \omega_{j}
\end{aligned}
$$

By using this formula, we can evaluate the scattering kernel. The Hartree-Fock terms are given by Eqs. (B1) and (B2). We obtain

$$
\begin{aligned}
\mathcal{W}_{H F}^{>}= & \int \prod_{j} d \mathbf{p}_{j} \delta\left(\mathfrak{E}(\mathbf{p})+\mathfrak{E}\left(\mathbf{p}_{1}\right)-\mathfrak{E}\left(\mathbf{p}_{2}\right)-\mathfrak{E}\left(\mathbf{p}_{3}\right)\right) \delta\left(\mathbf{p}+\mathbf{p}_{1}-\mathbf{p}_{2}-\mathbf{p}_{3}\right) \\
& \times\left[f^{>}(\mathbf{p}) f^{>}\left(\mathbf{p}_{1}\right) f^{<}\left(\mathbf{p}_{2}\right) f^{<}\left(\mathbf{p}_{3}\right)-f^{<}(\mathbf{p}) f^{<}\left(\mathbf{p}_{1}\right) f^{>}\left(\mathbf{p}_{2}\right) f^{>}\left(\mathbf{p}_{3}\right)\right] \mathrm{T}_{H F},
\end{aligned}
$$

where tr denotes the matrix trace and

$$
\begin{aligned}
\mathrm{T}_{H F}= & \operatorname{tr}\left\{\mathcal{M}(\mathbf{p}) \mathcal{M}\left(\mathbf{p}_{2}\right)\right\}\left[\operatorname{tr}\left\{\mathcal{M}\left(\mathbf{p}_{1}\right) \mathcal{M}\left(\mathbf{p}_{3}\right)\right\}+\operatorname{tr}\left\{\mathcal{M}^{\perp}\left(\mathbf{p}_{3}\right) \mathcal{M}^{\perp}\left(\mathbf{p}_{1}\right)\right\}\right] \\
& +\operatorname{tr}\left\{\mathcal{M}(\mathbf{p}) \mathcal{M}^{\perp}\left(\mathbf{p}_{1}\right)\right\} \operatorname{tr}\left\{\mathcal{M}^{\perp}\left(\mathbf{p}_{2}\right) \mathcal{M}\left(\mathbf{p}_{3}\right)\right\}+2 \operatorname{tr}\left\{\mathcal{M}(\mathbf{p}) \mathbf{X}_{F}\right\} \\
= & \left(\mathfrak{u}_{p} \mathfrak{u}_{1} \mathfrak{u}_{2} \mathfrak{u}_{3}+\mathfrak{v}_{p} \mathfrak{u}_{1} \mathfrak{v}_{2} \mathfrak{u}_{3}+\mathfrak{v}_{p} \mathfrak{u}_{1} \mathfrak{u}_{2} \mathfrak{v}_{3}+\mathfrak{v}_{p} \mathfrak{v}_{1} \mathfrak{v}_{2} \mathfrak{u}_{3}+\mathfrak{u}_{p} \mathfrak{v}_{1} \mathfrak{v}_{2} \mathfrak{v}_{3}\right)^{2} \\
\mathrm{X}_{F}= & \mathcal{M}\left(\mathbf{p}_{2}\right) \mathcal{M}\left(\mathbf{p}_{1}\right) \mathcal{M}\left(\mathbf{p}_{3}\right)+\mathcal{M}^{\perp}\left(\mathbf{p}_{1}\right) \mathcal{M}^{\perp}\left(\mathbf{p}_{2}\right) \mathcal{M}\left(\mathbf{p}_{3}\right)+\mathcal{M}\left(\mathbf{p}_{2}\right) \mathcal{M}^{\perp}\left(\mathbf{p}_{3}\right) \mathcal{M}^{\perp}\left(\mathbf{p}_{1}\right) .
\end{aligned}
$$

In the same way, we group together the other terms describing collisions between the noncondensed cloud and the condensate phase. We obtain

$$
\begin{aligned}
\mathcal{W}_{c n}^{>}= & -c 16 \pi n_{c} \int \prod_{j} d \mathbf{p}_{j} \delta\left(\mathfrak{E}(\mathbf{p})+\mathfrak{E}\left(\mathbf{p}_{1}\right)-\mathfrak{E}\left(\mathbf{p}_{2}\right)\right) \delta\left(\mathbf{p}+\mathbf{p}_{1}-\mathbf{p}_{2}\right) \\
& \times\left[f^{>}(\mathbf{p}) f^{>}\left(\mathbf{p}_{1}\right) f^{<}\left(\mathbf{p}_{2}\right)-f^{<}(\mathbf{p}) f^{<}\left(\mathbf{p}_{1}\right) f^{>}\left(\mathbf{p}_{2}\right)\right] \mathbf{T}_{c n}\left(\mathfrak{E}\left(\mathbf{p}_{1}\right), \mathfrak{E}\left(\mathbf{p}_{2}\right)-\mathfrak{E}\left(\mathbf{p}_{1}\right), \mathfrak{E}\left(\mathbf{p}_{2}\right)\right) \\
& -c 8 \pi n_{c} \int \prod_{j} d \mathbf{p}_{j} \delta\left(\mathfrak{E}(\mathbf{p})-\mathfrak{E}\left(\mathbf{p}_{1}\right)-\mathfrak{E}\left(\mathbf{p}_{2}\right)\right) \delta\left(\mathbf{p}-\mathbf{p}_{1}-\mathbf{p}_{2}\right) \\
& \times\left[f^{>}(\mathbf{p}) f^{<}\left(\mathbf{p}_{1}\right) f^{<}\left(\mathbf{p}_{2}\right)-f^{<}(\mathbf{p}) f^{>}\left(\mathbf{p}_{1}\right) f^{>}\left(\mathbf{p}_{2}\right)\right] \mathbf{T}_{c n}\left(\mathfrak{E}\left(\mathbf{p}_{2}\right)+\mathfrak{E}\left(\mathbf{p}_{1}\right), \mathfrak{E}\left(\mathbf{p}_{1}\right), \mathfrak{E}\left(\mathbf{p}_{2}\right)\right),
\end{aligned}
$$

where

$$
\begin{aligned}
\mathrm{T}_{c n}\left(\mathfrak{E}_{3}, \mathfrak{E}_{1}, \mathfrak{E}_{2}\right)= & 2 \operatorname{tr}\left\{\mathcal{M}(\mathbf{p}) \mathcal{M}\left(\mathbf{p}_{2}\right) \mathcal{M}^{\perp}\left(\mathbf{p}_{3}\right) \mathfrak{J}+\mathcal{M}(\mathbf{p}) \mathfrak{J} \mathcal{M}^{\perp}\left(\mathbf{p}_{2}\right) \mathcal{M}\left(\mathbf{p}_{3}\right)+\mathcal{M}(\mathbf{p}) \mathcal{M}\left(\mathbf{p}_{2}\right) \mathfrak{J} \mathcal{M}\left(\mathbf{p}_{3}\right)\right\} \\
& +\operatorname{tr}\left\{\mathcal{M}(\mathbf{p}) \mathcal{M}\left(\mathbf{p}_{2}\right)\right\} \operatorname{tr}\left\{\mathfrak{J}\left[\mathcal{M}^{\perp}\left(\mathbf{p}_{3}\right)+\mathcal{M}\left(\mathbf{p}_{3}\right)\right]\right\}+\operatorname{tr}\{\mathcal{M}(\mathbf{p}) \mathfrak{J}\} \operatorname{tr}\left\{\mathcal{M}^{\perp}\left(\mathbf{p}_{2}\right) \mathcal{M}\left(\mathbf{p}_{3}\right)\right\} \\
= & \left(\mathfrak{u}_{1} \mathfrak{u}_{2} \mathfrak{u}_{3}+\mathfrak{v}_{1} \mathfrak{v}_{2} \mathfrak{v}_{3}+\mathfrak{u}_{1} \mathfrak{v}_{2} \mathfrak{v}_{3}+\mathfrak{v}_{1} \mathfrak{u}_{2} \mathfrak{v}_{3}-\mathfrak{u}_{1} \mathfrak{v}_{2} \mathfrak{u}_{3}-\mathfrak{v}_{1} \mathfrak{u}_{2} \mathfrak{u}_{3}\right)^{2}
\end{aligned}
$$

and $\mathfrak{E}_{i} \equiv \mathfrak{E}\left(\mathbf{p}_{i}\right), \mathfrak{u}_{i} \equiv \mathfrak{u}\left(\mathbf{p}_{i}\right), \mathfrak{v}_{i} \equiv \mathfrak{v}\left(\mathbf{p}_{i}\right)$. 


\section{APPENDIX C: CONDENSED-NONCONDENSED SCATTERING KERNEL}

We derive the explicit form of the scattering kernel used in the simulations. We first consider the collision term $\mathcal{W}_{n c}$. From Eq. (20), we see that $\mathcal{W}_{n c}$ has the following form:

$$
\begin{aligned}
\mathfrak{S} & =\int \mathcal{F}\left(\mathbf{p}_{0}, \mathbf{p}_{1}, \mathbf{p}_{2}\right) \delta\left(\mathfrak{E}\left(p_{0}\right) \pm \mathfrak{E}\left(p_{1}\right)-\mathfrak{E}\left(p_{2}\right)\right) \delta\left(\mathbf{p}_{0} \pm \mathbf{p}_{1}-\mathbf{p}_{2}\right) d \mathbf{p}_{1} d \mathbf{p}_{2}, \\
& =\int \mathcal{F}\left(\mathbf{p}_{0}, \mathbf{p}_{1}, \mathbf{p} \pm \mathbf{p}_{1}\right) \delta\left(\mathfrak{E}\left(p_{0}\right) \pm \mathfrak{E}\left(p_{1}\right)-\mathfrak{E}\left(\left|\mathbf{p}_{0} \pm \mathbf{p}_{1}\right|\right)\right) p_{1}^{2} \sin \left(\theta_{01}\right) d \varphi_{01} d \theta_{01} d p_{1},
\end{aligned}
$$

where the function $\mathcal{F}$ depends on the distance from the center of the sphere, i.e., the projection of the momentum along and orthogonal to the radial direction $\hat{\rho}$. In order to simplify the previous integral, it is convenient to define some spherical coordinate directions. We define by $(\hat{\boldsymbol{\rho}}, \hat{\boldsymbol{\theta}}, \hat{\boldsymbol{\phi}})$ the reference frame with the $z$ direction along the radius of the sphere and define by $\left(\hat{\boldsymbol{\rho}}_{\mathbf{p}_{i}}, \hat{\boldsymbol{\theta}}_{\mathbf{p}_{i}}, \hat{\boldsymbol{\phi}}_{\mathbf{p}_{i}}\right)$ the reference frame with the $z$ direction along $\mathbf{p}_{i}$. Moreover, $\theta_{i j}$ are the angles between $\mathbf{p}_{i}$ and $\mathbf{p}_{j}$ and $\varphi_{01}$ is the spin angle of $\mathbf{p}_{1}$ on the reference $\left(\hat{\boldsymbol{\rho}}_{\mathbf{p}_{0}}, \hat{\boldsymbol{\theta}}_{\mathbf{p}_{0}}, \hat{\boldsymbol{\varphi}}_{\mathbf{p}_{0}}\right)$. By using the monotonicity of $\mathfrak{E}$, we have that (considering $\theta_{01}$ as variable) the equation

$$
\mathfrak{E}\left(p_{3}\right)=\mathfrak{E}\left(p_{0}\right) \pm \mathfrak{E}\left(p_{1}\right),
$$

with $\mathbf{p}_{3}=\mathbf{p}_{0} \pm \mathbf{p}_{1}$, has one and only one solution if $\mathfrak{E}\left(p-p_{1}\right) \leqslant \mathfrak{E}(p) \pm \mathfrak{E}\left(p_{1}\right) \leqslant \mathfrak{E}\left(p+p_{1}\right)$. More precisely, since $\mathfrak{E}$ depends only on the modulus of the momentum, the previous equation defines a class of solutions parameterized by the angle $\varphi_{01}$ (when $\mathbf{p}_{1}$ rotates around $\mathbf{p}_{0}$, Eq. $(\mathrm{C} 1)$ always gives the same solution $)$. We denote this solution by $\mathbf{p}_{1}^{ \pm}=\mathbf{p}_{1}^{ \pm}\left[\widetilde{\theta^{ \pm}}\left(p_{0}, p_{1}\right), \varphi_{01}\right] . \widetilde{\theta^{ \pm}}$is given by $\left|\mathbf{p}_{0} \pm \mathbf{p}_{1}\right|^{2}=p_{0}^{2}+p_{1}^{2} \pm 2 p_{0} p_{1} \cos \left(\widetilde{\theta^{ \pm}}\right)$. Explicitly,

$$
\cos \left(\widetilde{\theta^{ \pm}}\right)= \pm \frac{2 m\left[\sqrt{\left(\mathfrak{E}\left(p_{0}\right)+\mathfrak{E}\left(p_{1}\right)\right)^{2}+g^{2} n_{c}^{2}}-\frac{m}{2} \mathbf{V}_{c}^{2}-\mu+2 g n_{c}\right]-p_{0}^{2}-p_{1}^{2}}{2 p_{0} p_{1}}
$$

and $\mathbf{p}_{1}^{ \pm}=p_{1} \cos \left(\widetilde{\theta^{ \pm}}\right) \hat{\boldsymbol{\rho}}_{\mathbf{p}_{0}}+p_{1} \sin \left(\widetilde{\theta^{ \pm}}\right)\left[\cos \left(\varphi_{01}\right) \hat{\boldsymbol{\theta}}_{\mathbf{p}_{0}}+\sin \left(\varphi_{01}\right) \hat{\boldsymbol{\varphi}}_{\mathbf{p}_{0}}\right]$. These expressions can be written in the original basis by using $\hat{\boldsymbol{\rho}}_{\mathbf{p}_{0}}=\hat{\boldsymbol{\rho}} p_{0} \cos \left(\theta_{0}\right)+\hat{\boldsymbol{\theta}} p_{0} \sin \left(\theta_{0}\right) \cos \left(\varphi_{0}\right)+\hat{\boldsymbol{\varphi}} p_{0} \sin \left(\theta_{0}\right) \sin \left(\varphi_{0}\right)$ and similar expressions. This leads to

$$
\begin{aligned}
\mathfrak{S} & =\frac{m}{p} \int_{0}^{2 \pi} \int_{0}^{E^{ \pm}\left(p_{0}\right)} \mathcal{F}\left(\mathbf{p}_{0}, \mathbf{p}_{1}^{ \pm}, \mathbf{p}_{3}^{ \pm}\right) \frac{p_{1}}{\left.\sqrt{1+\left[\frac{g n_{c}}{\mathfrak{E}\left(p_{0}\right) \pm \mathfrak{E}\left(p_{1}\right)}\right.}\right]^{2}} \vartheta d \varphi_{01} d p_{1}, \\
\vartheta & =\Theta_{H}\left[\mathfrak{E}_{B}(p) \pm \mathfrak{E}_{B}\left(p_{1}\right)-\mathfrak{E}_{B}\left(p-p_{1}\right)\right] \Theta_{H}\left[\mathfrak{E}_{B}\left(p+p_{1}\right)-\mathfrak{E}_{B}(p) \mp \mathfrak{E}_{B}\left(p_{1}\right)\right],
\end{aligned}
$$

where $\Theta_{H}$ denotes the Heaviside step function, $E^{+}=\infty, E^{-}=\mathfrak{E}(p)$, and we use

$$
\left.\frac{\partial \mathfrak{E}\left(\left|\mathbf{p} \pm \mathbf{p}_{1}\right|\right)}{\partial \theta}\right|_{\mathfrak{E}\left(\left|\mathbf{p} \pm \mathbf{p}_{1}\right|\right)=\mathfrak{E}(p) \pm \mathfrak{E}\left(p_{1}\right)}=\mp \frac{p p_{1} \sin (\theta)}{m} \sqrt{1+\left[\frac{g n_{c}}{\mathfrak{E}(p) \pm \mathfrak{E}\left(p_{1}\right)}\right]^{2}}
$$

Finally, by using the fact that the function $\mathfrak{E}(p)$ is convex and invertible, we can show that $\vartheta=1$ always.

The Boltzmann scattering collision term between two noncondensed particles $\mathcal{W}_{H F}$ has a more standard form and has already been studied by various authors (see, for example, Ref. [31]). For the sake of completeness, we review here the main steps of the expression that can be directly implemented. $\mathcal{W}_{H F}$ has the following form:

$$
W=\int F\left[\mathfrak{E}\left(p_{1}\right), \mathfrak{E}\left(p_{2}\right), \mathfrak{E}\left(p_{3}\right), \mathfrak{E}\left(p_{4}\right)\right] \delta\left(\mathbf{p}_{1}+\mathbf{p}_{2}-\mathbf{p}_{3}-\mathbf{p}_{4}\right) \delta\left[\mathfrak{E}\left(p_{1}\right)+\mathfrak{E}\left(p_{2}\right)-\mathfrak{E}\left(p_{3}\right)-\mathfrak{E}\left(p_{4}\right)\right] d \mathbf{p}_{2} d \mathbf{p}_{3} d \mathbf{p}_{4}
$$

Integration with respect to $\mathbf{p}_{4}$ gives

$$
\begin{aligned}
W= & 2 \pi \int F\left[\mathfrak{E}\left(p_{1}\right), \mathfrak{E}\left(p_{2}\right), \mathfrak{E}\left(\left|\mathbf{p}_{1}-\widetilde{\mathbf{p}}\right|\right), \mathfrak{E}\left(\left|\widetilde{\mathbf{p}}+\mathbf{p}_{2}\left(p_{2}, \vartheta_{2}\right)\right|\right)\right] \\
& \times \delta\left[\mathfrak{E}\left(p_{1}\right)+\mathfrak{E}\left(p_{2}\right)-\mathfrak{E}\left(\left|\mathbf{p}_{1}-\widetilde{\mathbf{p}}\right|\right)-\mathfrak{E}\left(\left|\widetilde{\mathbf{p}}+\mathbf{p}_{2}\left(p_{2}, \vartheta_{2}\right)\right|\right)\right] p_{2}^{2} \sin \left(\vartheta_{2}\right) d \vartheta_{2} d p_{2} d \widetilde{\mathbf{p}}
\end{aligned}
$$

where we denoted $\widetilde{\mathbf{p}}=\mathbf{p}_{1}-\mathbf{p}_{3}$ and $\vartheta_{2}$ is the angle between $\widetilde{\mathbf{p}}$ and $\mathbf{p}_{2}$. The integration with respect to $\vartheta_{2}$ gives

$$
\begin{aligned}
W= & 4 \pi^{2} \int F\left[\mathfrak{E}\left(p_{1}\right), \mathfrak{E}\left(p_{2}\right), \mathfrak{E}\left(\left|\mathbf{p}_{1}-\widetilde{\mathbf{p}}\right|\right), \mathfrak{E}\left(p_{1}\right)+\mathfrak{E}\left(p_{2}\right)-\mathfrak{E}\left(\left|\mathbf{p}_{1}-\widetilde{\mathbf{p}}\right|\right)\right] \Theta_{H}\left[\mathfrak{E}\left(p_{1}\right)+\mathfrak{E}\left(p_{2}\right)-\mathfrak{E}\left(\left|\mathbf{p}_{1}-\widetilde{\mathbf{p}}\right|\right)-\mathfrak{E}\left(\tilde{p}-p_{2}\right)\right] \\
& \times \Theta_{H}\left[\mathfrak{E}\left(\widetilde{p}+p_{2}\right)-\mathfrak{E}\left(p_{1}\right)-\mathfrak{E}\left(p_{2}\right)+\mathfrak{E}\left(\left|\mathbf{p}_{1}-\widetilde{\mathbf{p}}\right|\right)\right] \frac{p_{2} \widetilde{p} \sin (\widetilde{\vartheta})}{2\left|\frac{d \mathfrak{E}}{d p^{2}}\right|_{\mathfrak{E}=\widetilde{E}\left(p_{1}, p_{2}, \widetilde{\vartheta}\right)} d p_{2} d \widetilde{p} d \widetilde{\vartheta}}
\end{aligned}
$$


We make the substitution $\widetilde{\vartheta} \rightarrow p$ with $p^{2}=\left|\mathbf{p}_{1}-\widetilde{\mathbf{p}}\right|^{2}=p_{1}^{2}+\widetilde{p}^{2}-2 \widetilde{p} p_{1} \cos (\widetilde{\vartheta})$ and we change the integration order between $p$ and $\tilde{p}$,

$$
\begin{aligned}
W(\mathbf{p})= & \frac{2 \pi^{2}}{p} \int_{0}^{\infty} \int_{0}^{\infty} d p_{2} d p_{1} \int_{\left|p-p_{1}\right|}^{p+p_{1}} d p_{3} F\left[\mathfrak{E}_{B}\left(p_{2}\right), \mathfrak{E}_{B}\left(p_{1}\right), \mathfrak{E}_{B}(p)+\mathfrak{E}_{B}\left(p_{2}\right)-\mathfrak{E}_{B}\left(p_{1}\right)\right] \\
& \times \Theta_{H}\left[\mathfrak{E}_{B}(p)+\mathfrak{E}_{B}\left(p_{2}\right)-\mathfrak{E}_{B}\left(p_{1}\right)-\mathfrak{E}_{B}\left(p_{3}-p_{2}\right)\right] \Theta_{H}\left[\mathfrak{E}_{B}\left(p_{3}+p_{2}\right)-\mathfrak{E}_{B}(p)-\mathfrak{E}_{B}\left(p_{2}\right)+\mathfrak{E}_{B}\left(p_{1}\right)\right] \\
& \times \frac{p_{2} p_{1}}{\left|\frac{d \mathfrak{E}_{B}}{d p^{2}}\right|_{\mathfrak{E}_{B}^{-1}\left[\mathfrak{E}_{B}(p)+\mathfrak{E}_{B}\left(p_{2}\right)-\mathfrak{E}_{B}\left(p_{1}\right)\right]} \mid}
\end{aligned}
$$

Some calculations show that in the energy variables $E_{i}=\mathfrak{E}\left(p_{i}\right), W$ takes the form

$$
\begin{aligned}
W(E)= & m^{3} \frac{4 \pi^{2}}{\mathfrak{E}_{B}^{-1}(E)} \int_{0}^{\infty} d E_{1} \int_{0}^{E+E_{1}} d E_{2} \\
& \times F\left[E, E_{1}, E_{2}, E+E_{1}-E_{2}\right] \frac{\left(\widetilde{p}_{M}-\widetilde{p}_{m}\right)}{\sqrt{1+\left(\frac{g n_{c}}{E+E_{1}-E_{2}}\right)^{2}}} \frac{1}{\sqrt{1+\left(\frac{g n_{c}}{E_{2}}\right)^{2}}} \frac{1}{\sqrt{1+\left(\frac{g n_{c}}{E_{1}}\right)^{2}}},
\end{aligned}
$$

where

$$
\begin{aligned}
& \widetilde{p}_{m}\left(E, E_{1}, E_{2}\right)=\max \left[\left|\mathfrak{E}^{-1}(E)-\mathfrak{E}^{-1}\left(E_{2}\right)\right|, \quad\left|\mathfrak{E}^{-1}\left(E_{1}\right)-\mathfrak{E}^{-1}\left(E+E_{1}-E_{2}\right)\right|\right], \\
& \widetilde{p}_{M}\left(E, E_{1}, E_{2}\right)=\min \left[\mathfrak{E}^{-1}(E)+\mathfrak{E}^{-1}\left(E_{2}\right), \quad \mathfrak{E}^{-1}\left(E_{1}\right)+\mathfrak{E}^{-1}\left(E+E_{1}-E_{2}\right)\right] .
\end{aligned}
$$

[1] A. L. Fetter, J. Low Temp. Phys. 129, 263 (2002).

[2] P. M. Platzman and A. P. Mills, Phys. Rev. B 49, 454 (1994).

[3] S. T. Beliaev, Sov. Phys. JETP 7, 289 (1958).

[4] T. D. Doan, H. T. Cao, D. B. Tran Thoai, and H. Haug, Phys. Rev. B 72, 085301 (2005).

[5] O. M. Schmitt, D. B. Tran Thoai, L. Banyai, P. Gartner, and H. Haug, Phys. Rev. Lett. 86, 3839 (2001).

[6] A. Griffin, T. Nikuni, and E. Zaremba, Bose Condensed Gases at Finite Temperatures (Cambridge University Press, Cambridge, UK, 2009).

[7] L. Banyai, P. Gartner, O. M. Schmitt, and H. Haug, Phys. Rev. B 61, 8823 (2000).

[8] A. Bezett and P. B. Blakie, Phys. Rev. A 79, 023602 (2009).

[9] J. E. Williams and A. Griffin, Phys. Rev. A 64, 013606 (2001).

[10] H. Shi and A. Griffin, Phys. Rep. 304, 1 (1998).

[11] F. Tassone and Y. Yamamoto, Phys. Rev. B 59, 10830 (1999).

[12] A. J. Allen, C. F. Barenghi, N. P. Proukakis, and E. Zaremba, arXiv: $1206.0145 \mathrm{v} 1$.

[13] T. R. Kirkpatrick and J. R. Dorfman, J. Low Temp. Phys. 58, 301 (1985).

[14] T. R. Kirkpatrick and J. R. Dorfman, Phys. Rev. A 28, 2576 (1983).

[15] M. Imamovic-Tomasovic and A. Griffin, J. Low Temp. Phys. 122, 617 (2001).

[16] O. Morandi, Phys. Rev. B 80, 024301 (2009).

[17] O. Morandi, Quantum Phase-Space Transport and Applications to the Solid State Physics, Some Applications of Quantum Mechanics, edited by M. R. Pahlavani (InTech, New York, 2012).

[18] O. Morandi and F. Schuerrer, J. Phys. A: Math. Theor. 44, 265301 (2011).

[19] L. P. Kadanoff and G. Baym, Quantum Statistical Mechanics (Benjamin, New York, 1962).

[20] S. A. Moskalenko and D. W. Snoke, Bose-Einstein Condensation of Excitons and Biexcitons: And Coherent Nonlinear Optics
With Excitons (Cambridge University Press, Cambridge, UK, 2000).

[21] B. Capogrosso-Sansone, S. Giorgini, S. Pilati, L. Pollet, N. Prokof'ev, B. Svistunov, and M. Troyer, New J. Phys. 12, 043010 (2010).

[22] L. P. Pitaevskii and S. Stringari, Bose-Einstein Condensation (Oxford University Press, New York, 2003).

[23] E. D. Gust and L. E. Reichl, arXiv:1202.3418v1.

[24] W. Bao, D. Jaksch, and P. A. Markowich, J. Comput. Phys. 187, 318 (2003).

[25] M. Edwards and K. Burnett, Phys. Rev. A 51, 1382 (1995).

[26] B. Jackson and E. Zaremba, Phys. Rev. A 66, 033606 (2002).

[27] E. Zaremba, T. Nikuni, and A. Griffin, J. Low Temp. Phys. 116, 277 (1999).

[28] M. J. Davis, C. W. Gardiner, and R. J. Ballagh, Phys. Rev. A 62, 033600 (2000).

[29] M. J. Bijlsma, E. Zaremba, and H. T. C. Stoof, Phys. Rev. A 62, 063609 (2000).

[30] K. Oda, T. Miyakawa, H. Yabu, and T. Suzuki, J. Phys. Soc. Jpn. 70, 1549 (2001).

[31] D. W. Snoke and J. P. Wolfe, Phys. Rev. B 39, 4030 (1989).

[32] D. V. Semikoz and I. I. Tkachev, Phys. Rev. Lett. 74, 3093 (1995).

[33] C. W. Gardiner, M. D. Lee, R. J. Ballagh, M. J. Davis, and P. Zoller, Phys. Rev. Lett. 81, 5266 (1998).

[34] V. E. Zakharov, Zh. Eksp. Teor. Fiz. 51, 688 (1966).

[35] Yu. Kagan, B. V. Svistunov, and G. V. Shlyapnikov, Sov. Phys. JETP 75, 387 (1992).

[36] M. D. Lee and C. W. Gardiner, Phys. Rev. A 62, 033606 (2000).

[37] H.-J. Miesner, D. M. Stamper-Kurn, M. R. Andrews, D. S. Durfee, S. Inouye, and W. Ketterle, Science 279, 1005 (1998).

[38] A. A. Abrikosov, L. P. Gor'kov, and I. Ye. Dzyaloshinskii, Quantum Field Theoretical Methods in Statistical Physics (Pergamon, Oxford, 1965). 